\title{
A HIDDEN POPULATION OF MASSIVE STARS WITH CIRCUMSTELLAR SHELLS DISCOVERED WITH THE SPITZER SPACE TELESCOPE
}

\author{
Stefanie Wachter ${ }^{1}$, Jon C. Mauerhan ${ }^{1}$, Schuyler D. Van Dyk ${ }^{1}$, D. W. Hoard ${ }^{1}$, Stella KafKa ${ }^{2,4}$, and \\ PATRICK W. MORRIS ${ }^{3}$ \\ ${ }^{1}$ Spitzer Science Center, California Institute of Technology, MS 220-6, Pasadena, CA 91125, USA; \\ wachter@ipac.caltech.edu, mauerhan@ipac.caltech.edu, vandyk@ipac.caltech.edu, hoard@ipac.caltech.edu \\ ${ }^{2}$ Department of Terrestrial Magnetism, Carnegie Institution of Washington, 5241 Broad Branch Road NW, Washington, DC 20015, USA; skafka@dtm.ciw.edu \\ ${ }^{3}$ NASA Herschel Science Center, California Institute of Technology, Pasadena, CA 91125, USA; pmorris@ipac.caltech.edu \\ Received 2010 January 7; accepted 2010 March 22; published 2010 April 26
}

\begin{abstract}
We have discovered a large number of circular and elliptical shells at $24 \mu \mathrm{m}$ around luminous central sources with MIPS on board the Spitzer Space Telescope. Our archival follow-up effort has revealed $90 \%$ of these circumstellar shells to be previously unknown. The majority of the shells is only visible at $24 \mu \mathrm{m}$, but many of the central stars are detected at multiple wavelengths from the mid- to the near-IR regime. The general lack of optical counterparts, however, indicates that these sources represent a population of highly obscured objects. We obtained optical and near-IR spectroscopic observations of the central stars and find most of these objects to be massive stars. In particular, we identify a large population of sources that we argue represents a narrow evolutionary phase, closely related or identical to the luminous blue variable stage of massive stellar evolution.

Key words: circumstellar matter - ISM: bubbles - stars: early-type - stars: emission-line, Be - stars: mass-loss stars: Wolf-Rayet
\end{abstract}

Online-only material: extended figure

\section{INTRODUCTION}

The Spitzer Space Telescope has provided us with an unprecedented view of the mid-IR sky in terms of both resolution and sensitivity. Invariably, the opening of every new wavelength window carries with it the potential for the discovery of previously unrecognized phenomena and source populations, which have gone unnoticed at other wavelengths. Motivated by our discovery of an unusual well-defined elliptical shell-lacking a central energizing source and only visible at $24 \mu \mathrm{m}$-in some of the first data from the Spitzer mission (Morris et al. 2006), we executed a search for similar objects that might shed light on its still puzzling origin and nature.

We utilized the publicly available $24 \mu \mathrm{m}$ data products (delivery Version 3.0) of the MIPSGAL Legacy project (Carey et al. 2009) obtained with MIPS (Rieke et al. 2004). We conducted our search by eye, inspecting all the images of the inner $248 \mathrm{deg}^{2}$ of the Galactic plane covered by the MIPSGAL survey. Although there was no prediction of whether $24 \mu \mathrm{m}$ shells would be rare or common occurrences, we were still surprised by our discovery of a large number of prominent, more or less ring-shaped $24 \mu \mathrm{m}$ objects: we find over 200 such shells in the MIPSGAL survey area. Unlike the shell that originally motivated the search, however, the majority of the objects have some indication of a central source, at times quite luminous even at $24 \mu \mathrm{m}$.

A follow-up investigation utilizing the complementary data from the GLIMPSE Spitzer Legacy project (Benjamin et al. 2003) which covers the same survey area with the IRAC 3.5-8.0 $\mu \mathrm{m}$ bands, as well as the Two Micron All Sky Survey (2MASS) and the Digitized Sky Survey (DSS), shows that about $60 \%$ of these shells are only detected at $24 \mu \mathrm{m}$ (see Figure 1).

\footnotetext{
4 Visiting Astronomer, Cerro Tololo Inter-American Observatory, which is operated by the Association of Universities for Research in Astronomy, Inc. under cooperative agreement with the National Science Foundation.
}

This is somewhat unusual, as we generally expect a strong emission component due to polycyclic aromatic hydrocarbons (PAHs) at $8 \mu \mathrm{m}$ if we are simply observing warm dust continuum emission. In the case of the shell discussed by Morris et al. (2006), the lack of $8 \mu \mathrm{m}$ detection is explained by a pure line emission spectrum with a dominant [O IV] $25.89 \mu \mathrm{m}$ line. We speculate that some of our $24 \mu \mathrm{m}$-only shells might exhibit similar mid-IR spectral characteristics.

Ring-like shell morphology is observed frequently in planetary nebulae or in material associated with a variety of massive stars, such as Wolf-Rayet (W-R) stars and red and blue supergiants (RSGs and BSGs; Chu 1991). So far, most known circumstellar rings and shells have largely been studied using narrow band optical filters (e.g., $\mathrm{H} \alpha$, [O III]) and, despite being labeled as ring-shaped, many of these nebulae appear quite amorphous. For our Spitzer-discovered shell sources, a SIMBAD search within $2^{\prime}$ of the shell locations revealed that most ( $90 \%$ of the shells, $80 \%$ of the central sources) of these objects have not been previously studied. We present here the results of our efforts to determine the nature of the central stars that produce the $24 \mu \mathrm{m}$ shells and to characterize the properties of this substantial population of new sources.

\section{SOURCE SELECTION}

Identifying a region of $24 \mu \mathrm{m}$ emission as a more or less symmetrical shell is necessarily a subjective undertaking. In order to compile the most homogeneous sample possible, we have selected a subsample of our shells for dedicated follow-up observations, based on the following criteria:

1. The $24 \mu \mathrm{m}$ shell has a symmetric (circular or elliptical) shape with a well-defined boundary.

2 . The presence of a likely central source, detected at a minimum of two different wavelengths among the $24 \mu \mathrm{m}$, $8.0 \mu \mathrm{m}, 3.6 \mu \mathrm{m}, 2 \mathrm{MASS} K s$ and $J$ images. 
The second criterion is particularly tricky, since we are dealing with the crowded fields in the Galactic plane and, hence, a relatively high a priori probability for chance superpositions. In order to classify a given source as a "likely" central source, we first fitted each shell by eye with a circle or ellipse, then searched for sources within $1^{\prime \prime}$ of the geometric center of the fitted shape. Shells with bright $24 \mu \mathrm{m}$ central sources represent the most reliable cases, since the overall source density at this wavelength is significantly lower than in the shorter wavelength bands. In other cases, the central source identification is more tentative, and we comment on this for individual sources in the Appendix. Larger shells have greater uncertainties in the determination of the geometric center purely from the error inherent in the fitting process. In addition, the literature certainly contains examples of shells excited by sources that are significantly offset from the geometrical center (e.g., Whitehead et al. 1988; Siebenmorgen et al. 1998).

Our list of targets is presented in Table 1. For each shell, we have indicated the 2MASS designation of the likely central source, the approximate angular size of the shell, the presence of the central source counterpart at various wavelengths, and whether the $24 \mu \mathrm{m}$ shell is also detected at $8 \mu \mathrm{m}$. We searched for known objects at the position of the central sources in SIMBAD, using a $2^{\prime}-5^{\prime}$ radius depending on the size of each shell. The results are listed in the last column of Table 1, as well as the results of our IR and optical spectroscopic follow-up of the central sources.

In order to facilitate the correlation of central source properties with the morphological characteristics of the shells, Spitzer 24 and $8 \mu \mathrm{m}, 2$ MASS $K s$ - and DSS $R$-band images of the shells are displayed in Figure 1 (in its entirety available only in electronic format). The assumed central source is marked.

\section{OBSERVATIONS OF THE CENTRAL SOURCES}

While some of the central sources from Table 1 can be matched to objects in SIMBAD, the vast majority has not been observed before. It is impossible to determine the nature of the central sources based solely on archival photometric data, since the extinction-both intrinsic to the source and between us and the source-and the distance to the shells are unknown. Hence, we obtained optical and IR spectroscopy of the central sources of these newly discovered shells to identify their nature. The spectroscopic data obtained is indicated by "IR" for infrared and "OPT" for optical in Table 1, respectively.

\subsection{Near-infrared Spectroscopy}

Near-infrared spectroscopy of a number of the central stars of our shells was obtained on 2009 June 23-25 UT with the Ohio State Infrared Imager and Spectrograph (OSIRIS; Depoy et al. 1993) on the Southern Observatory for Astrophysical Research (SOAR) 4.1m telescope. Our low-resolution spectra $(R \sim 1200)$ were obtained using the $1^{\prime \prime}$ cross-dispersed slit and the f3 camera which covers the $J, H$, and $K$ bands simultaneously. Spectra were acquired in an "ABBA" nodding sequence for sky subtraction. We utilized sky spectra for wavelength calibration. Data reduction and spectral extraction and calibration were performed in the standard manner using IRAF. For telluric correction, spectra of bright A0V standard stars $(K s \approx 6-7 \mathrm{mag})$ were obtained. The IDL program xtellcor (Vacca et al. 2003) was used, which removes model $\mathrm{HI}$ absorption lines from the A0V spectra before application to the science data. Observing conditions were photometric on the first night, but strongly affected by variable clouds during the following two nights, which limited the observations to the brighter targets in our source list. Note that no $J$-band spectra could be extracted for star nos. 4, 5, 6a, 6b, 8a, 14, 20, 22, 29, and 44.

The IR spectrum for star no. 52 was obtained with the Palomar Hale 200" telescope and the TripleSpec (TSPEC) near-infrared spectrograph on 2009 July 14 UT. TSPEC provides simultaneous coverage of the $J, H$, and $K$ bands $(\lambda=1.0-2.4 \mu \mathrm{m})$ and produces a moderate resolution spectrum $(R \approx 2500-2700)$ through a $1^{\prime \prime} \times 30^{\prime \prime}$ slit. Spectra were obtained utilizing an ABBA telescope nodding sequence for sky subtraction and bad-pixel suppression. Wavelength calibration was performed using the $\mathrm{OH}$ emission lines in the sky spectra. The A0V star HR 6958 was observed as a standard, and a telluric spectrum was applied to the science data using the program xtellcor.

\subsection{Optical Spectroscopy}

For the limited number of sources with optical counterparts, optical spectroscopy was obtained with the 200" Hale telescope at Palomar Observatory on 2008 September 3 and 4 UT using the Double Spectrograph. This low to medium resolution grating spectrograph uses a dichroic to split the light into separate red and blue channels which are observed simultaneously. The blue side CCD has $15 \mu \mathrm{m}$ pixels and a scale of 0.389 pixel $^{-1}$, the red side CCD $24 \mu \mathrm{m}$ pixels with a scale of 0.468 pixel $^{-1}$. We used the 316 lines $\mathrm{mm}^{-1}$ grating in first order, dichroic D55 and a slit width of $1^{\prime \prime}$. The resulting spectra cover 4000-5600 $\AA$ and 5800-8300 $\AA$ with a dispersion of 2.0 and $2.4 \AA$ pixel $^{-1}$ on the blue and red sides, respectively, and have a resolution of 5-7 ̊. For wavelength calibration, FeAr (on the blue side) and $\mathrm{HeNeAr}$ (on the red side) arc lamp spectra were obtained at each new telescope position. Bias and flat-field corrections to the raw spectrum images were performed with IRAF. Various routines within the IRAF packages twodspec and onedspec were used to extract and wavelength/flux calibrate the spectra. Both nights were photometric with variable seeing. Observations of spectrophotometric standard stars were carried out, however, these were mainly used to remove the instrumental response function. A signal-to-noise ratio of 40-100 was achieved in the final spectra.

\section{SPECTRAL CLASSIFICATION}

\subsection{IR Spectra}

At first glance, our central source IR spectra can be roughly divided into two main groups: those that show emission lines or early spectral type features and those that exhibit absorption features indicative of late-type stars.

\subsubsection{Late-type Stars}

For the late-type stars, we determined the spectral type and luminosity class based on the strength of the $\mathrm{CO}$ bandhead at $2.29 \mu \mathrm{m}$, following the methodology of Figer et al. (2006) and Davies et al. (2007). Both ways provided consistent results. We also compared the appearance of the spectra to those from the Infrared Telescope Facility (IRTF) spectral atlas (Rayner et al. 2009). In addition, we performed the same analysis for selected spectra from the atlas (of stars with known spectral type and luminosity class) as an independent check for the validity of our measurements. We estimate that our derived spectral types are accurate to within \pm 1 subtype. The results are listed in Table 2 
Table 1

Shell Data

\begin{tabular}{|c|c|c|c|c|c|}
\hline Num & 2MASS J Designation & $\begin{array}{c}\text { Radius } \\
(\operatorname{arcmin})\end{array}$ & $\begin{array}{c}\text { Central src } \\
24 / 8 / 3.6 / 1.1\end{array}$ & $\begin{array}{l}\text { Shell at } \\
8 \mu \mathrm{m} \text { ? }\end{array}$ & Comments $^{\mathrm{a}}$ \\
\hline 1 & $11441803-6245210$ & $0.53 \times 0.35$ & $\mathrm{y} / \mathrm{y} / \mathrm{y} / \mathrm{y}$ & no & IRAS 11419-6228; Oe/WN (IR), this work \\
\hline 2 & $13012188-6244131$ & $0.94 \times 1.01$ & $\mathrm{y} / \mathrm{y} / \mathrm{y} / \mathrm{y}$ & yes & \\
\hline 3 & $13104384-6317457$ & 0.45 & $\mathrm{y} / \mathrm{y} / \mathrm{y} / \mathrm{y}$ & no & Oe/WN (IR), this work \\
\hline 4 & $14211002-6138199$ & $1.08 \times 2.38$ & $\mathrm{y} / \mathrm{y} / \mathrm{y} / \mathrm{y}$ & yes & IRAS 14173-6124; M6 III/K5 I (IR), this work \\
\hline 5 & $15075621-5818250$ & $0.50 \times 0.42$ & $\mathrm{y} / \mathrm{y} / \mathrm{y} / \mathrm{y}$ & no & M0 III/K0 I (IR), this work \\
\hline $6 a$ & $15092899-5847430$ & $0.49 \times 0.80$ & $\mathrm{y} / \mathrm{y} / \mathrm{y} / \mathrm{y}$ & yes & M6 III/K5 I (IR), this work \\
\hline $6 b$ & $15092951-5847551$ & & $\mathrm{y} / \mathrm{y} / \mathrm{y} / \mathrm{y}$ & & M5 III/K4 I (IR), this work \\
\hline 7 & $15255986-5704403$ & 0.17 & $\mathrm{y} / \mathrm{y} / \mathrm{y} / \mathrm{y}$ & yes & \\
\hline $8 \mathrm{a}$ & $15330809-5612200$ & 0.66 & $\mathrm{y} / \mathrm{y} / \mathrm{y} / \mathrm{y}$ & no & K1 III/G7 I (IR), this work \\
\hline $8 b$ & $15330663-5612219$ & & $\mathrm{y} / \mathrm{y} / \mathrm{y} / \mathrm{y}$ & & M5 III/K4 I (IR), this work \\
\hline 9 & $15352652-5604123$ & 1.07 & $\mathrm{y} / \mathrm{y} / \mathrm{y} / \mathrm{y}$ & no & WN7 (Mauerhan et al. 2009) \\
\hline 10 & $15455914-5332325$ & 0.70 & $\mathrm{y} / \mathrm{y} / \mathrm{y} / \mathrm{y}$ & no & IRAS 15421-5323; WN9h (IR), this work \\
\hline $11 \mathrm{a}$ & $15484207-5507422$ & 1.57 & $\mathrm{y} / \mathrm{y} / \mathrm{y} / \mathrm{y}$ & no & $\mathrm{Be} / \mathrm{B}[\mathrm{e}] / \mathrm{LBV}(\mathbf{I R})$, this work \\
\hline $11 \mathrm{~b}$ & $15484210-5507542$ & & & & WC9 (IR), this work \\
\hline 12 & $15553788-5343402$ & $1.24 \times 2.42$ & $\mathrm{y} / \mathrm{y} / \mathrm{y} / \mathrm{y}$ & yes & IRAS 15517-5334: \\
\hline 13 & $15581378-5257513$ & 0.85 & $\mathrm{y} / \mathrm{y} / \mathrm{y} / \mathrm{y}$ & no & Oe/WN (IR), this work \\
\hline 14 & $16290377-4746264$ & 0.33 & $\mathrm{y} / \mathrm{y} / \mathrm{y} / \mathrm{y}$ & yes & IRAS $16254-4739 ; \mathrm{Be} / \mathrm{B}[\mathrm{e}] / \mathrm{LBV}$ (IR), this work \\
\hline 15 & $16313781-4814553$ & $0.31 \times 0.28$ & $\mathrm{n} / \mathrm{y} / \mathrm{y} / \mathrm{y}$ & no & IRAS 16278-4808: \\
\hline 16 & $16321298-4750358$ & 1.02 & $\mathrm{n} / \mathrm{y} / \mathrm{y} / \mathrm{y}$ & no & WN5b (Shara et al. 2009) \\
\hline 17 & $16364278-4656207$ & 0.30 & $\mathrm{y} / \mathrm{y} / \mathrm{y} / \mathrm{y}$ & no & $\mathrm{Be} / \mathrm{B}[\mathrm{e}] / \mathrm{LBV}(\mathbf{I R})$, this work \\
\hline 18 & $16431636-4600424$ & 0.95 & $\mathrm{y} / \mathrm{y} / \mathrm{y} / \mathrm{y}$ & no & IRAS 16396-4555, SS 73 63; Be (Pereira et al. 2003) \\
\hline 19 & $16461734-4508478$ & 0.18 & $\mathrm{y} / \mathrm{y} / \mathrm{y} / \mathrm{y}$ & no & \\
\hline 20 & $16493770-4535592$ & 1.48 & $\mathrm{y} / \mathrm{y} / \mathrm{y} / \mathrm{y}$ & no & $\mathrm{Be}(\mathbf{I R})$, this work \\
\hline 21 & $17051043-4053071$ & 0.25 & $\mathrm{n} / \mathrm{n} / \mathrm{y} / \mathrm{y}$ & yes & PN G345.4+00.1, IC 4637, Hen 2-193, WRAY 15-1607 \\
\hline 22 & $17072333-3956504$ & $0.43 \times 0.71$ & $\mathrm{y} / \mathrm{y} / \mathrm{y} / \mathrm{y}$ & yes & M1 I (IR), this work \\
\hline $23 \mathrm{a}$ & $17082913-3925076$ & $0.79 \times 0.73$ & $\mathrm{y} / \mathrm{y} / \mathrm{y} / \mathrm{y}$ & no & IRAS $17050-3921 ; \mathrm{Be} / \mathrm{B}[\mathrm{e}] / \mathrm{LBV}$ (IR), this work \\
\hline $23 b$ & $17082930-3925158$ & & $\mathrm{y} / \mathrm{y} / \mathrm{y} / \mathrm{y}$ & & M2 III/K2 I (IR), this work \\
\hline 24 & $17110094-3945174$ & 0.46 & $\mathrm{y} / \mathrm{y} / \mathrm{y} / \mathrm{y}$ & no & $\mathrm{Be} / \mathrm{B}[\mathrm{e}] / \mathrm{LBV}(\mathbf{I R})$, this work \\
\hline 25 & $17352942-3246562$ & $0.55 \times 0.47$ & $\mathrm{y} / \mathrm{y} / \mathrm{y} / \mathrm{y}$ & no & \\
\hline $26 \mathrm{a}$ & $17370371-3147467$ & $1.09 \times 0.92$ & $\mathrm{y} / \mathrm{y} / \mathrm{y} / \mathrm{y}$ & yes & M0 III/K0 I (IR), this work \\
\hline $26 b$ & $17370394-3147349$ & & $\mathrm{y} / \mathrm{y} / \mathrm{y} / \mathrm{y}$ & & G9 III (IR), this work \\
\hline $27 \mathrm{a}$ & $17374754-3137333$ & 0.51 & $\mathrm{y} / \mathrm{y} / \mathrm{y} / \mathrm{y}$ & yes & \\
\hline $27 b$ & $17374730-3137370$ & & $\mathrm{n} / \mathrm{y} / \mathrm{y} / \mathrm{y}$ & & \\
\hline $28 \mathrm{a}$ & $17391899-3124239$ & 0.55 & $\mathrm{y} / \mathrm{y} / \mathrm{y} / \mathrm{y}$ & no & OB (IR), this work \\
\hline $28 b$ & $17391913-3124142$ & & $\mathrm{y} / \mathrm{y} / \mathrm{y} / \mathrm{y}$ & & M6 III/K5 I (IR), this work \\
\hline 29 & $17413543-3006389$ & 1.0 & $\mathrm{y} / \mathrm{y} / \mathrm{y} / \mathrm{y}$ & yes & WRAY 17-96, Hen 3-1453, LBV candidate, (IR) \\
\hline 30 & $17421401-2955360$ & 0.28 & $\mathrm{y} / \mathrm{y} / \mathrm{y} / \mathrm{y}$ & yes & \\
\hline 31 & $17433908-2431525$ & 0.24 & $\mathrm{n} / \mathrm{y} / \mathrm{y} / \mathrm{y}$ & yes & PN G003.5+02.7 \\
\hline 32 & $17435981-3028384$ & $1.04 \times 0.76$ & $\mathrm{y} / \mathrm{y} / \mathrm{y} / \mathrm{y}$ & no & star M2:; Be (IR), this work \\
\hline 33 & $17481403-2800531$ & $1.54 \times 1.97$ & $\mathrm{y} / \mathrm{y} / \mathrm{y} / \mathrm{y}$ & yes & HD 316285 , Hen 3-1482, WRAY 15-1777, LBV candidate \\
\hline 34 & $17493539-2649302$ & $0.68 \times 0.79$ & $\mathrm{y} / \mathrm{y} / \mathrm{y} / \mathrm{y}$ & yes & \\
\hline 35 & $18005762-2433467$ & 0.17 & $\mathrm{n} / \mathrm{y} / \mathrm{y} / \mathrm{y}$ & yes & IRAS $17578-2433$ \\
\hline 36 & $18022233-2238002$ & 0.42 & $\mathrm{y} / \mathrm{y} / \mathrm{y} / \mathrm{y}$ & yes & Oe/WN (IR), this work \\
\hline 37 & $18035667-2256000$ & 0.21 & $\mathrm{y} / \mathrm{n} / \mathrm{y} / \mathrm{y}$ & yes & \\
\hline 38 & $18070516-2015163$ & 0.36 & $\mathrm{y} / \mathrm{y} / \mathrm{y} / \mathrm{y}$ & no & \\
\hline 39 & $18133121-1856431$ & 0.28 & $\mathrm{n} / \mathrm{y} / \mathrm{y} / \mathrm{y}$ & no & Be: (OPT), this work \\
\hline 40 & $18233420-1525076$ & 0.27 & $\mathrm{n} / \mathrm{y} / \mathrm{y} / \mathrm{y}$ & no & \\
\hline 41 & $18284156-1027056$ & 0.77 & $\mathrm{y} / \mathrm{y} / \mathrm{y} / \mathrm{y}$ & no & \\
\hline 42 & $18321739-0916139$ & 0.14 & $\mathrm{n} / \mathrm{n} / \mathrm{y} / \mathrm{y}$ & no & \\
\hline 43 & $18333954-0807084$ & 0.55 & $\mathrm{y} / \mathrm{y} / \mathrm{y} / \mathrm{y}$ & no & G7 I (IR), this work \\
\hline 44 & $18335528-0658386$ & 0.74 & $\mathrm{y} / \mathrm{y} / \mathrm{y} / \mathrm{y}$ & yes & G 024.73+00.69, V481 Sct, LBV, (IR, OPT) \\
\hline 45 & $18393224-0544204$ & $1.39 \times 1.04$ & $\mathrm{y} / \mathrm{y} / \mathrm{y} / \mathrm{y}$ & yes & G 026.47+00.02, 2MASS J18393224-0544204, LBV candidate, (IR) \\
\hline 46 & $18415965-0515409$ & 0.43 & $\mathrm{y} / \mathrm{y} / \mathrm{y} / \mathrm{y}$ & yes & $\mathrm{Be} / \mathrm{B}[\mathrm{e}] / \mathrm{LBV}(\mathbf{I R})$, this work \\
\hline 47 & $18420630-0348224$ & 1.67 & $\mathrm{y} / \mathrm{y} / \mathrm{y} / \mathrm{y}$ & no & \\
\hline 48 & $18420827-0351029$ & 1.16 & $\mathrm{y} / \mathrm{y} / \mathrm{y} / \mathrm{y}$ & no & \\
\hline 49 & $18422247-0504300$ & 0.47 & $\mathrm{y} / \mathrm{y} / \mathrm{y} / \mathrm{y}$ & no & IRAS $18397-0507$ \\
\hline 50 & $18424692-0313172$ & 0.29 & $\mathrm{n} / \mathrm{y} / \mathrm{y} / \mathrm{y}$ & yes & PN G029.0+00.4; WN6 (OPT), this work \\
\hline 51 & $18455593-0308297$ & 0.40 & $\mathrm{y} / \mathrm{y} / \mathrm{y} / \mathrm{y}$ & yes & \\
\hline 52 & $18492733-0104207$ & 1.8 & $\mathrm{y} / \mathrm{y} / \mathrm{y} / \mathrm{y}$ & no & WN7 (IR, OPT), this work \\
\hline 53 & $18503980+0004453$ & 0.33 & $\mathrm{n} / \mathrm{y} / \mathrm{y} / \mathrm{y}$ & no & IRAS $18481+0001$ \\
\hline 54 & $18510295-0058242$ & 1.07 & $\mathrm{y} / \mathrm{y} / \mathrm{y} / \mathrm{y}$ & no & \\
\hline 55 & $18530582+0011358$ & 0.24 & $\mathrm{n} / \mathrm{y} / \mathrm{y} / \mathrm{y}$ & no & \\
\hline 56 & $19011669+0355108$ & 0.52 & $\mathrm{y} / \mathrm{y} / \mathrm{y} / \mathrm{y}$ & yes & IRAS 18588+0350: \\
\hline 57 & $19042098+0600001$ & 0.74 & $\mathrm{y} / \mathrm{y} / \mathrm{y} / \mathrm{y}$ & no & $\mathrm{F} / \mathrm{G}(\mathbf{O P T})$, this work \\
\hline 58 & $19325284+1742303$ & 0.78 & $\mathrm{y} / \mathrm{y} / \mathrm{y} / \mathrm{y}$ & no & B0e-B5e (OPT), this work \\
\hline
\end{tabular}


Table 1

(Continued)

\begin{tabular}{lccccc}
\hline \hline Num & 2MASS J Designation & $\begin{array}{c}\text { Radius } \\
(\operatorname{arcmin})\end{array}$ & $\begin{array}{c}\text { Central src } \\
24 / 8 / 3.6 / 1.1\end{array}$ & $\begin{array}{c}\text { Shell at } \\
8 \mu \mathrm{m} ?\end{array}$ & Comments $^{\mathrm{a}}$ \\
\hline $59 \mathrm{a}$ & $19385510+2127550$ & $0.89 \times 0.49$ & $\mathrm{y} / \mathrm{y} / \mathrm{y} / \mathrm{y}$ & yes & M2-M4 (OPT), this work \\
$59 \mathrm{~b}$ & $19385569+2127483$ & & & & no \\
60 & $19443759+2419058$ & 0.53 & $\mathrm{y} / \mathrm{y} / \mathrm{y} / \mathrm{y}$ & no & IRAS 19425+2411, HBHA 2203-01; Be (OPT, this work) \\
61 & $19444295+2311337$ & 0.46 & $\mathrm{y} / \mathrm{y} / \mathrm{y} / \mathrm{y}$ & $\mathrm{n} / / / \mathrm{y}-\mathrm{B} 5$ (OPT), this work \\
$62 \mathrm{a}$ & $19550232+2917178$ & 0.42 & $\mathrm{y} / / / \mathrm{y}$ & & PN G065.9+00.5, NGC 6842, Hen 2-451 \\
$62 \mathrm{~b}$ & $19550249+2917198$ & & & \\
\hline
\end{tabular}

Note. ${ }^{\text {a }}$ For detailed notes on each object see the Appendix.
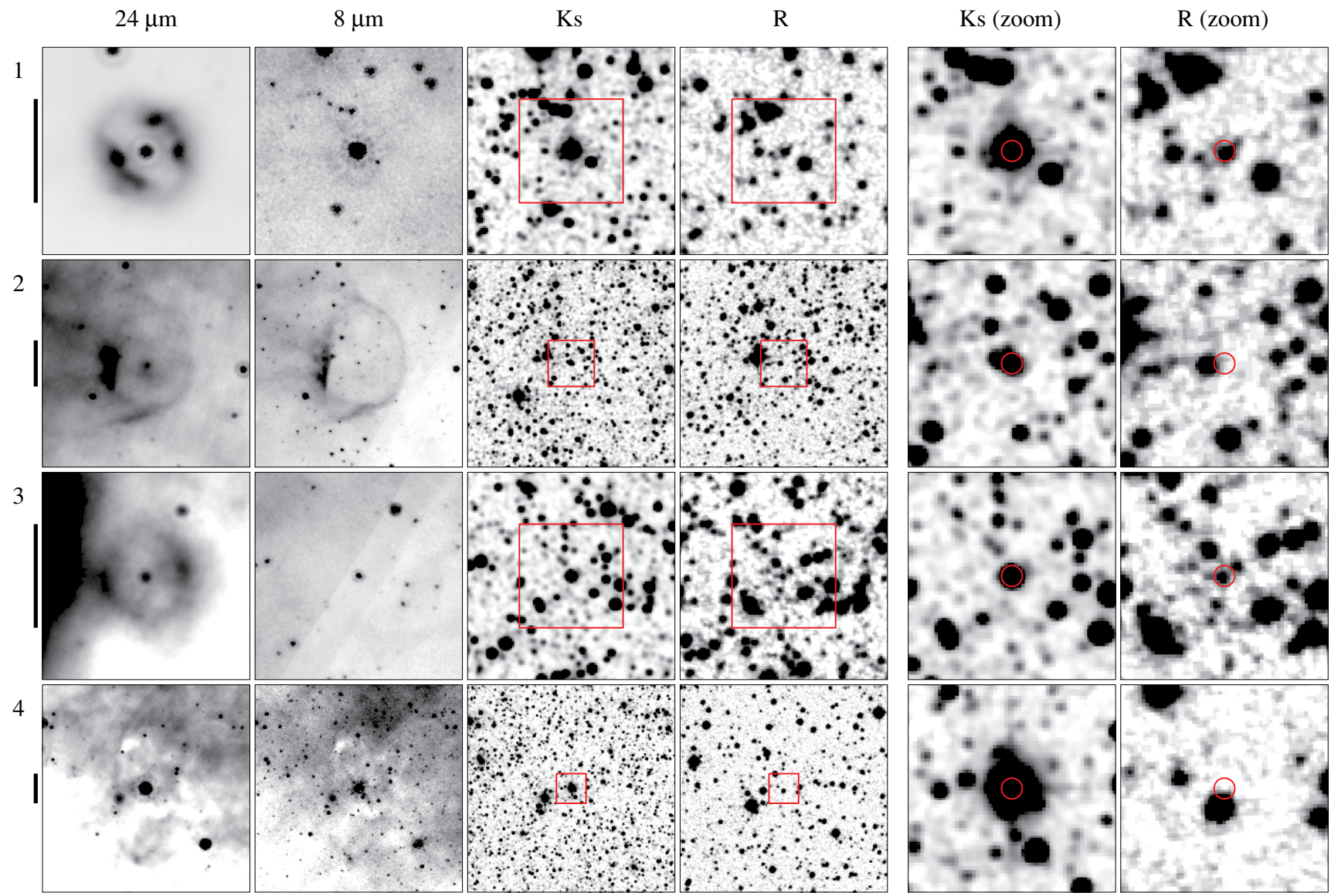

Figure 1. MIPS $24 \mu \mathrm{m}$ images of our newly discovered circumstellar shells together with the corresponding IRAC $8 \mu \mathrm{m}, 2 \mathrm{MASS} K s$, and DSS $R$-band images. North is up and east is to the left. A $1^{\prime}$ scale bar is indicated on the left-hand side of each row of panels. The assumed central source for each shell is marked. The $K s$ - and $R$-band images are displayed at two different scales; the two rightmost panels have a field of view of $30^{\prime \prime} \times 30^{\prime \prime}$.

(An extended version of this figure is available in the online journal.)

and a selection of our late-type spectra are displayed in Figure 2. Note that several sources show $\mathrm{Br} \gamma$ in emission.

We caution that veiling of the absorption features by a hot dust continuum could affect our spectral type determination. This would lead to an underestimate of our measured equivalent width (EW), and hence the true spectral types of our sources would be later than those we derived. In order to investigate the presence of circumstellar dust, we plotted the 2MASS colors of the stars from Table 2 in a near-IR color-color diagram (not shown). Typically, IR excess due to circumstellar material will cause a shift to redder $H-K s$ colors relative to the unreddened stellar loci. We find that all of our stars fall on the red (super)giant reddening line within the photometric uncertainties. The most deviant point corresponds to star no. $23 \mathrm{~b}$, which is unlikely to produce the associated shell as discussed below.
As indicated in Table 2, each CO bandhead EW measurement corresponds to a solution for both giants and supergiants, and we are unable to break the degeneracy solely based on our spectra. Hence, we investigated whether photometric information could provide additional constraints to distinguish between these possibilities. For each source, we first calculated the total (interstellar and circumstellar) $A_{V}$ by comparing its 2MASS photometry (provided in Table 3) with the unreddened $J-K$ colors of the appropriate spectral type from Cox (2000), utilizing the reddening law by Cardelli et al. (1989). Finally, we derived the distances and the physical sizes of the surrounding shells for the two possible luminosity classes, respectively. These results are also listed in Table 2 .

For all stars, the derived $A_{V}$ exceeds the average $1 \mathrm{mag} \mathrm{kpc}^{-1}$ value by several factors, implying that the line of sight to these 

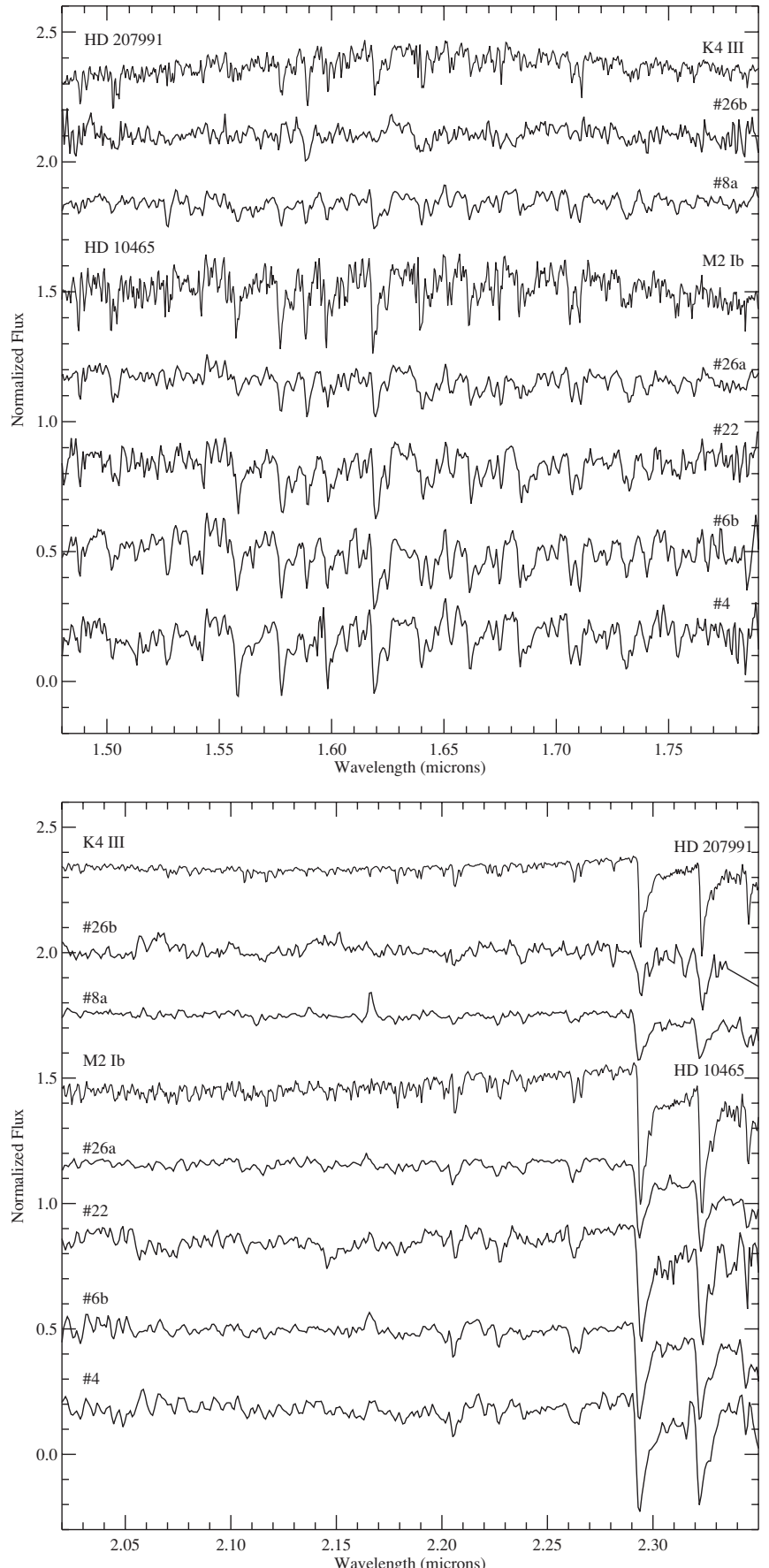

Figure 2. $H$-and $K$-band spectra of a sample of shell central sources indicating a late-type star. The spectra have been normalized and offset from each other for display purposes. For comparison, the spectra of HD 207991 (K4 III) and HD 10465 (M2Ib) obtained from the IRTF spectral library (Rayner et al. 2009) are also shown.

sources intersects localized areas of high extinction. Minimizing this excess extinction would indicate the supergiant case for all sources. However, for star no. 26b, we favor the giant scenario, since the distance of $>23 \mathrm{kpc}$ for the supergiant case results in an average extinction of only $0.4 \mathrm{mag} \mathrm{kpc}^{-1}$ and the largest physical shell size of the sample. We derive and list these parameters for all of the sources for completeness, however, star nos. 8b, 23b, and $28 \mathrm{~b}$ are probably not producing their respective observed shells. These particular stars are members of apparent doubles, where the center of the fitted shell geometry coincides with the other component of the double. While we cannot exclude the
Table 2

Late-type Sources

\begin{tabular}{|c|c|c|c|c|}
\hline Source & Type & $\begin{array}{c}A_{V} \\
(\mathrm{mag}) \\
\end{array}$ & $\begin{array}{c}d \\
(\mathrm{kpc})\end{array}$ & $\begin{array}{l}\text { Size } \\
(\mathrm{pc})\end{array}$ \\
\hline \multirow[t]{2}{*}{4} & M6 III & 29.8 & 1.1 & $0.34 \times 0.75$ \\
\hline & K5 I & 32.5 & 2.6 & $0.80 \times 1.77$ \\
\hline \multirow[t]{2}{*}{5} & M0 III & 7.8 & 1.2 & $0.17 \times 0.14$ \\
\hline & K0 I & 10.4 & 6.1 & $0.89 \times 0.75$ \\
\hline \multirow[t]{2}{*}{$6 a$} & M6 III & 12.0 & 3.2 & $0.46 \times 0.75$ \\
\hline & K5 I & 14.7 & 7.6 & $1.08 \times 1.76$ \\
\hline \multirow[t]{2}{*}{$6 \mathrm{~b}$} & M5 III & 13.8 & 2.5 & $0.36 \times 0.58$ \\
\hline & K4 I & 16.7 & 7.6 & $1.09 \times 1.77$ \\
\hline \multirow[t]{2}{*}{$8 a$} & K1 III & 14.4 & 0.6 & 0.11 \\
\hline & G7 I & 15.3 & 9.6 & 1.85 \\
\hline \multirow[t]{2}{*}{$8 b^{a}$} & M5 III & 12.2 & 4.1 & 0.78 \\
\hline & K4 I & 15.0 & 12.5 & 2.39 \\
\hline 22 & M1 I & 8.6 & 4.3 & $0.53 \times 0.88$ \\
\hline \multirow[t]{2}{*}{$23 b^{a}$} & M2 III & 13.1 & 2.7 & $0.63 \times 0.58$ \\
\hline & K2 I & 15.7 & 11.6 & $2.67 \times 2.47$ \\
\hline \multirow[t]{2}{*}{$26 a$} & M0 III & 10.2 & 2.1 & $0.67 \times 0.56$ \\
\hline & K0 I & 12.8 & 11.0 & $3.50 \times 2.96$ \\
\hline \multirow[t]{2}{*}{$26 \mathrm{~b}$} & G9 III & 9.6 & 1.4 & $0.44 \times 0.37$ \\
\hline & G5 I & 10.4 & 23.7 & $7.51 \times 6.34$ \\
\hline \multirow[t]{2}{*}{$28 b^{a}$} & M6 III & 18.2 & 5.3 & 0.86 \\
\hline & K5 I & 20.9 & 12.6 & 2.01 \\
\hline \multirow[t]{2}{*}{43} & K1 III & 24.8 & 0.3 & 0.44 \\
\hline & G7 I & 25.6 & 4.6 & 0.74 \\
\hline
\end{tabular}

Note. ${ }^{\text {a }}$ Probably not producing the shell, see the text for details.

possibility that the off-center source is responsible for the shell, it appears more likely that the central source is producing the shell, in particular in the case of nos. $23 \mathrm{~b}$ and $28 \mathrm{~b}$, where the central source was found to be a hot star (see the following section).

Most of our knowledge about large-scale circumstellar shells around red giants and supergiants dates back to the IRAS $60 \mu \mathrm{m}$ surveys of Stencel et al. (1989) and Young et al. (1993). Young et al. (1993) surveyed 512 red giant stars, $15 \%$ of which were found to have shells with radii ranging from 0.1 to $4.6 \mathrm{pc}$. The average shell radius was $0.74 \mathrm{pc}$, with radii of $>2 \mathrm{pc}$ being quite rare. Stencel et al. (1989) conducted a similar survey for 111 red supergiants, $25 \%$ of which showed evidence for shells with radii between 0.1 and $4 \mathrm{pc}$ (most clustered between 0.1 and $1.5 \mathrm{pc}$ ). Our shells have very similar size characteristics, which is somewhat surprising given that (1) our shells were observed at $24 \mu \mathrm{m}$ and one might expect size differences between the shells at various wavelengths, and (2) the resolution of the IRAS $60 \mu \mathrm{m}$ images was quite limiting, such that these surveys were biased toward detecting only the largest shells of any underlying distribution.

\subsubsection{Emission Line/Early-type Stars}

Most of our IR spectra with emission lines resemble those of the so-called "transition objects," massive stars of type Of, $\mathrm{WNL}, \mathrm{Be}, \mathrm{B}[\mathrm{e}]$ and luminous blue variable (LBV), thought to span the evolutionary stages between $\mathrm{O}$ and W-R stars (Morris et al. 1996). The classification of these stars based on IR spectra is notoriously difficult. We find that our spectra separate fairly cleanly into two distinct groups simply based on the appearance of their spectral features. When considering commonalities, we give less weight to the appearance of the He I $2.058 \mu \mathrm{m}$ line, since it is highly variable due to its susceptibility to optical depth effects. 
Table 3

Shell Central Source Photometry

\begin{tabular}{|c|c|c|c|c|c|c|c|}
\hline Num & 2MASS $J$ & 2MASS $H$ & 2MASS $K s$ & $3.6 \mu \mathrm{m}^{\mathrm{a}}$ & $4.5 \mu \mathrm{m}^{\mathrm{a}}$ & $5.8 \mu \mathrm{m}^{\mathrm{a}}$ & $8.0 \mu \mathrm{m}^{\mathrm{a}}$ \\
\hline 1 & $9.999(23)$ & $8.720(31)$ & $7.775(23)$ & $6.982(26)$ & $\cdots$ & $6.175(37)$ & $5.813(30)$ \\
\hline 2 & $15.090(66)$ & $12.459(75)$ & $11.221(36)$ & $10.281(31)$ & $10.250(42)$ & $10.001(36)$ & $10.085(33)$ \\
\hline 3 & $12.271(23)$ & $11.004(20)$ & $10.186(19)$ & $9.516(31)$ & $9.065(41)$ & $8.824(28)$ & $8.450(22)$ \\
\hline 4 & $12.719(48)$ & $8.618(55)$ & $6.448(15)$ & $5.151(112)$ & $\ldots$ & $4.288(29)$ & $4.169(29)$ \\
\hline 5 & $9.282(20)$ & $7.815(21)$ & $6.959(13)$ & $6.600(61)$ & $6.144(57)$ & $5.900(37)$ & $5.882(33)$ \\
\hline $6 a$ & $10.061(19)$ & $7.905(39)$ & $6.776(7)$ & $6.697(74)$ & $\cdots$ & $5.731(33)$ & $5.594(26)$ \\
\hline $6 b$ & $10.870(23)$ & $8.520(41)$ & $7.311(9)$ & $6.617(46)$ & $6.482(46)$ & $6.142(35)$ & $6.020(27)$ \\
\hline 7 & $15.759(94)$ & $13.628(77)$ & $12.390(57)$ & $11.929(309)$ & 11.851(194) & $11.280(97)$ & $\ldots$ \\
\hline $8 \mathrm{a}$ & $11.678(28)$ & $9.688(20)$ & $8.573(19)$ & $7.572(36)$ & $7.274(47)$ & $7.043(32)$ & $6.890(27)$ \\
\hline $8 b$ & $11.472(21)$ & $9.218(20)$ & $8.189(29)$ & $7.510(47)$ & $7.592(46)$ & $7.330(34)$ & $7.228(23)$ \\
\hline 9 & $13.841(35)$ & $12.386(39)$ & $11.457(27)$ & $10.583(49)$ & $10.120(59)$ & $9.843(47)$ & $9.490(31)$ \\
\hline 10 & $14.576(34)$ & $12.217(36)$ & $10.874(21)$ & $9.447(64)$ & $9.023(55)$ & $8.620(48)$ & $8.399(29)$ \\
\hline $11 \mathrm{a}$ & $7.929(23)$ & $6.725(45)$ & $5.977(13)$ & $\ldots$ & $5.142(68)$ & $4.943(26)$ & $4.783(27)$ \\
\hline $11 \mathrm{~b}$ & $11.185(25)$ & $9.708(28)$ & $8.490(21)$ & $7.203(145)$ & $6.632(42)$ & $6.239(37)$ & $6.024(28)$ \\
\hline 12 & $12.380(25)$ & $8.637(17)$ & $6.603(17)$ & $5.215(182)$ & $\cdots$ & $3.923(25)$ & $3.969(33)$ \\
\hline 13 & $13.774(25)$ & $11.369(29)$ & $9.984(27)$ & $8.652(44)$ & $8.185(39)$ & $7.854(37)$ & $7.650(34)$ \\
\hline 14 & $8.668(27)$ & 7.141(43) & $6.188(15)$ & $6.749(106)$ & $5.626(63)$ & $5.076(31)$ & $4.735(23)$ \\
\hline 15 & $>16.567$ & $12.595(25)$ & $10.281(18)$ & $8.173(46)$ & $7.462(40)$ & $7.107(30)$ & $6.854(30)$ \\
\hline 16 & $14.998(52)$ & $12.697(33)$ & $11.340(26)$ & $10.049(49)$ & $9.514(44)$ & $9.196(88)$ & $8.988(46)$ \\
\hline 17 & 11.711(18) & $7.889(39)$ & $5.820(15)$ & $4.915(41)$ & $4.519(34)$ & $4.046(17)$ & $4.054(15)$ \\
\hline 18 & $6.258(17)$ & $5.077(45)$ & $4.205(33)$ & $\ldots$ & $\cdots$ & $\cdots$ & $\cdots$ \\
\hline 19 & $15.380(102)$ & $11.867(30)$ & $9.847(21)$ & $8.091(36)$ & $7.562(41)$ & $7.252(36)$ & $7.148(32)$ \\
\hline 20 & $7.242(17)$ & $6.086(33)$ & $5.422(17)$ & $\ldots$ & $5.022(55)$ & $4.518(32)$ & $4.336(19)$ \\
\hline 21 & $11.364(61)$ & $11.343(91)$ & $10.964(63)$ & $\ldots$ & $\ldots$ & $\ldots$ & $\ldots$ \\
\hline 22 & $7.009(9)$ & $5.325(13)$ & $4.635(13)$ & $\ldots$ & $\cdots$ & $4.096(28)$ & $4.109(23)$ \\
\hline $23 a$ & $10.492(21)$ & $8.219(51)$ & 7.111(19) & $6.463(87)$ & $6.164(55)$ & $5.714(26)$ & $5.545(29)$ \\
\hline $23 b$ & $12.081(21)$ & $10.032(24)$ & $8.790(17)$ & $7.686(47)$ & $7.151(53)$ & $6.788(32)$ & $6.442(25)$ \\
\hline 24 & $9.728(21)$ & $8.041(33)$ & $6.963(17)$ & $6.673(154)$ & $6.075(88)$ & $5.239(37)$ & $4.884(23)$ \\
\hline 25 & 10.749(19) & $9.101(20)$ & $8.254(17)$ & $7.633(36)$ & $7.443(29)$ & $7.295(29)$ & $7.336(19)$ \\
\hline $26 a$ & $11.260(23)$ & $9.342(23)$ & $8.527(21)$ & $7.949(31)$ & $8.093(29)$ & $7.885(30)$ & $7.872(19)$ \\
\hline $26 b$ & $12.556(23)$ & $11.014(26)$ & $10.339(32)$ & $9.365(49)$ & $9.038(90)$ & $8.711(34)$ & $8.536(21)$ \\
\hline $27 \mathrm{a}$ & $11.771(34)$ & $9.248(38)$ & 7.788(9) & $6.838(42)$ & $6.191(45)$ & $5.957(28)$ & $5.834(22)$ \\
\hline $27 b$ & $13.701(6)$ & $10.588(41)$ & $8.464(49)$ & $8.095(99)$ & $8.316(89)$ & $7.747(42)$ & $7.921(38)$ \\
\hline $28 \mathrm{a}$ & $8.440(19)$ & $7.586(29)$ & $7.078(11)$ & $6.864(33)$ & $6.657(44)$ & $6.548(27)$ & $6.573(23)$ \\
\hline $28 b$ & $>12.898$ & $10.078(36)$ & $8.578(31)$ & $7.464(28)$ & $7.502(29)$ & $7.080(28)$ & $7.029(18)$ \\
\hline 29 & $6.707(13)$ & $5.520(33)$ & $4.796(11)$ & $\ldots$ & $\ldots$ & $\ldots$ & $\ldots$ \\
\hline 30 & $13.382(33)$ & $10.540(33)$ & $8.993(29)$ & 7.677(39) & $7.158(35)$ & $6.843(27)$ & $6.862(23)$ \\
\hline 31 & $13.822(51)$ & $12.685(44)$ & $12.382(43)$ & $12.222(52)$ & $12.265(76)$ & $11.915(98)$ & $12.346(134)$ \\
\hline 32 & $5.743(13)$ & $4.781(75)$ & $4.070(33)$ & $\cdots$ & $\cdots$ & $\ldots$ & $\cdots$ \\
\hline 33 & $4.817(33)$ & $4.227(75)$ & $3.712(234)$ & $\ldots$ & $\ldots$ & $\ldots$ & $\ldots$ \\
\hline 34 & $8.906(13)$ & $8.319(39)$ & $8.106(19)$ & $8.026(29)$ & $8.138(27)$ & $8.076(23)$ & $8.035(19)$ \\
\hline 35 & $12.714(15)$ & $10.840(21)$ & $9.790(20)$ & $8.856(30)$ & $8.494(28)$ & $8.294(23)$ & $8.432(53)$ \\
\hline 36 & $13.604(51)$ & $11.073(32)$ & $9.597(27)$ & $8.365(45)$ & $7.762(44)$ & $7.499(30)$ & $7.293(24)$ \\
\hline 37 & $>14.022$ & $13.333(100)$ & $12.930(64)$ & $12.489(98)$ & $12.227(186)$ & $\ldots$ & $\ldots$ \\
\hline \multirow[t]{2}{*}{38} & $14.867(42)$ & $12.671(42)$ & $11.162(27)$ & $9.904(44)^{\mathrm{b}}$ & $\cdots$ & $9.180(41)^{b}$ & $\cdots$ \\
\hline & & & & $9.913(26)^{\mathrm{c}}$ & $9.433(39)^{\mathrm{c}}$ & $9.153(28)^{\mathrm{c}}$ & $8.988(30)^{\mathrm{c}}$ \\
\hline 39 & 11.569(17) & $10.340(25)$ & $9.648(19)$ & $9.256(76)$ & $9.044(63)$ & $8.896(47)$ & $8.867(37)$ \\
\hline 40 & $>15.565$ & $14.487(71)$ & $13.733(73)$ & $13.034(78)$ & $13.001(124)$ & $\cdots$ & $\cdots$ \\
\hline 41 & $>14.189$ & $12.630(74)$ & $11.327(39)$ & $10.090(144)$ & $9.688(62)$ & $9.395(57)$ & $9.139(48)$ \\
\hline 42 & $>13.148$ & $12.703(64)$ & $12.400(41)$ & 11.852(96) & $11.583(106)$ & $11.648(178)$ & $\ldots$ \\
\hline 43 & $12.998(38)$ & $9.851(22)$ & $8.151(19)$ & $7.100(87)$ & $6.392(47)$ & $6.108(35)$ & $6.050(28)$ \\
\hline 44 & $8.363(17)$ & $6.836(35)$ & $5.920(15)$ & $4.464(71)$ & $4.462(60)$ & $3.935(32)$ & $4.001(35)$ \\
\hline 45 & 7.997(13) & $6.526(21)$ & $5.608(9)$ & $4.796(81)$ & $4.211(63)$ & $3.811(29)$ & $\ldots$ \\
\hline 46 & $7.960(5)$ & $6.534(13)$ & $5.684(19)$ & $\ldots$ & $\ldots$ & $4.608(27)$ & $4.351(21)$ \\
\hline 47 & $11.946(26)$ & $10.216(20)$ & $9.162(19)$ & $8.277(37)$ & $7.630(42)$ & $7.430(28)$ & $7.039(30)$ \\
\hline 48 & $11.845(28)$ & $10.260(29)$ & $9.267(24)$ & $8.425(38)$ & $7.876(49)$ & $7.667(32)$ & $7.196(28)$ \\
\hline 49 & $>13.635$ & $11.101(35)$ & $8.684(23)$ & $6.873(66)$ & $\cdots$ & $5.750(30)$ & $5.672(31)$ \\
\hline 50 & $13.508(24)$ & $12.834(27)$ & $12.325(26)$ & 11.693(64) & $11.245(102)$ & $11.063(85)$ & $11.035(164)$ \\
\hline 51 & $15.447(61)$ & $11.505(21)$ & $9.373(23)$ & $7.389(45)$ & $6.717(47)$ & $6.316(35)$ & $6.100(28)$ \\
\hline 52 & $10.943(25)$ & $10.068(32)$ & $9.473(23)$ & $8.850(52)$ & $8.455(37)$ & $8.297(41)$ & $7.981(23)$ \\
\hline 53 & $>16.395$ & $13.645(57)$ & $12.169(35)$ & $10.855(59)$ & $10.433(56)$ & $10.163(65)$ & $9.886(39)$ \\
\hline 54 & $11.875(37)$ & $10.141(46)$ & $9.130(38)$ & $8.146(53)$ & $7.721(54)$ & $7.481(35)$ & $7.169(26)$ \\
\hline 55 & $14.428(29)$ & $12.538(25)$ & $11.638(24)$ & $10.923(57)$ & $10.652(69)$ & $10.597(77)$ & $10.700(72)$ \\
\hline 56 & $15.225(49)$ & $11.661(22)$ & $9.693(19)$ & $8.080(36)$ & $7.644(47)$ & $7.248(33)$ & $7.184(32)$ \\
\hline 57 & $>14.783$ & $12.960(38)$ & $11.574(30)$ & $10.204(76)$ & $9.551(54)$ & $9.270(45)$ & $8.958(35)$ \\
\hline 58 & $10.207(17)$ & $9.306(14)$ & $8.806(14)$ & $8.478(39)$ & $8.379(47)$ & $8.234(40)$ & $8.199(29)$ \\
\hline
\end{tabular}


Table 3

(Continued)

\begin{tabular}{|c|c|c|c|c|c|c|c|}
\hline Num & 2MASS $J$ & 2MASS $H$ & 2MASS $K s$ & $3.6 \mu \mathrm{m}^{\mathrm{a}}$ & $4.5 \mu \mathrm{m}^{\mathrm{a}}$ & $5.8 \mu \mathrm{m}^{\mathrm{a}}$ & $8.0 \mu \mathrm{m}^{\mathrm{a}}$ \\
\hline $59 a$ & $16.489(183)$ & $14.667(140)$ & 13.461(71) & $11.825(54)$ & $11.204(62)$ & $10.624(60)$ & $9.180(33)$ \\
\hline $59 b$ & $9.860(21)$ & $8.387(31)$ & $7.912(5)$ & $7.535(60)$ & $7.800(44)$ & $7.547(32)$ & $7.511(30)$ \\
\hline 60 & $8.857(13)$ & $8.016(19)$ & $7.418(15)$ & $6.734(30)$ & $6.421(38)$ & $6.176(22)$ & $5.837(16)$ \\
\hline 61 & $9.916(15)$ & $9.375(27)$ & $9.096(23)$ & $8.863(33)$ & $8.889(48)$ & $8.728(31)$ & $8.769(32)$ \\
\hline $62 \mathrm{a}$ & $15.681(111)$ & $>14.462$ & $>14.007$ & $\cdots$ & $\cdots$ & $\cdots$ & $\cdots$ \\
\hline $62 b$ & $15.557(66)$ & $>14.253$ & $>13.680$ & $\cdots$ & $\cdots$ & $\cdots$ & $\cdots$ \\
\hline
\end{tabular}

Notes.

a All magnitudes from the GLIMPSE I/II/3D survey. Data in italics are from the less reliable archive deliveries.

b GLIMPSE I.

c GLIMPSE II.

The first group is composed of star nos. 1,3,10,13,36, and 52 . For star no. 36, we only have a $K$-band spectrum; $J$ and $H$ were too noisy to reliably identify features. All stars exhibit strong He I 1.70 and $2.112 \mu \mathrm{m}$, as well as prominent $\mathrm{HI}$ emission in all three bands (Figure 3). For all figures, the line identifications have been compiled from Morris et al. (1996), Figer et al. (1997), and Crowther et al. (2006). We have divided the second group into two subgroups, distinguished by the strength of the $\mathrm{HI}$ features. The spectra in group 2A (star nos. 23a, 24, 45, and 46) are still dominated by $\mathrm{H}$ I emission of similar strength as group 1 in the $J, H$, and $K$ band, but the striking difference with the spectra of group 1 lies in the strong Fe II and Mg II emission and weak or absent $\mathrm{He}$ I $(1.70,2.112 \mu \mathrm{m})$ features (Figure 4). In addition, [Fe II] is detected in all the stars except no. 45. Group 2B (star nos. 11a, 14, 17, 29, 32, and 44) is characterized by much weaker $\mathrm{H}$ I emission (or even weak $\mathrm{HI}$ absorption) compared to the first two groups. However, similar to the stars from group 2A, the spectra exhibit Fe II or Mg II emission lines (Figure 5). We regard group 2B as an extension of group 2A toward weaker $\mathrm{H}$ I features. In particular, star no. 29 could have been assigned to either group $2 \mathrm{~A}$ or $2 \mathrm{~B}$, and emphasizes the connection between the two groups. Star no. 32 is included in group 2B based on the similarities of its spectral features in the $H$ band and the presence of $\mathrm{Pa} \beta$ emission in the $J$ band; however, it has no obvious emission lines of Fe II, and the $K$ band is featureless. In SIMBAD, the source is classified as M2: by Raharto et al. (1984), but an M spectral type can be ruled out based on our IR spectrum, given the $\mathrm{H}$ I absorption spectrum in the $H$ band and the lack of $\mathrm{CO}$ absorption features in the $K$ band.

We inspected all of our spectra for the presence of the He II $2.189 \mu \mathrm{m}$ line, since this is one of the key lines in identifying W-R and $\mathrm{O}$ type stars. Only star nos. 10, 52, and $11 \mathrm{~b}$ (Figure 6) exhibit convincing He II $2.189 \mu \mathrm{m}$ emission. Here, care must be taken to not mistake He I $2.185 \mu \mathrm{m}$ emission for that of He II $2.189 \mu \mathrm{m}$. Our spectra have sufficient resolution to clearly distinguish between the two lines, e.g., star no. 1 shows He I 2.185 and not He II $2.189 \mu \mathrm{m}$ emission. The ratios of the EWs of certain emission lines in the $K$ band are commonly used as a W-R subtype diagnostic (e.g., see Figer et al. 1997, Crowther et al. 2006). For star nos. 52 and 10, we measure $\operatorname{EW}(\lambda 2.189 \mu \mathrm{m}) / \operatorname{EW}(\lambda 2.165 \mu \mathrm{m})=0.5$ and $<0.1$, respectively, which correspond to W-R subtypes of WN7 and WN9h. We also have optical spectra of star no. 52 which independently confirm its WN7 classification based on a different set of spectral criteria (see Section 4.2). For star no. 11b, we find $\mathrm{EW}$ (C IV 2.076)/EW (C III 2.110) <1, consistent with a WC9 subtype.
Unfortunately, no classification scheme based on the EW measurement of features exists for the remaining spectra, and we have to rely on comparison to the various spectral atlases, e.g., Hanson et al. (1996) and Morris et al. (1996), to classify these sources. The stars from our group 2 strongly resemble the spectra of LBV and Be/B[e] stars from Morris et al. (1996) shown in their Figures 5-7. The exception in that sample is the LBV He 3-519, which does not exhibit Fe II emission, but instead looks like the spectra of stars in our group 1. It is interesting to note that [Fe II] $1.644 \mu \mathrm{m}$ emission has been found to be a common property of the shell emission among LBVs with prominent nebulae, but has rarely been seen in the spectra of their central sources (Smith 2002). In contrast, we observe [Fe II] emission in about half the sources of group 2. Based on these characteristics, we tentatively classify the stars in our group 2 as $\mathrm{Be} / \mathrm{B}[\mathrm{e}] / \mathrm{LBV}$ candidates. This is supported by the fact that star nos. 29,44 , and 45 are identified as LBVs or LBV candidates in the literature (for more details see Section 4.1.3).

Among the stars of group 1, we have already classified source no. 52 as WN7 and no. 10 as a WN9h star. The $K$-band spectra of the remaining four stars in group 1 (nos. 1, 3, 13, and 36) closely match the appearance of the WN9 source, except for the presence of the He II $2.189 \mu \mathrm{m}$ line. Several studies have classified similar-looking spectra as WN9, despite the lack of He II $2.189 \mu \mathrm{m}$ emission. For example, Figer et al. (1999) show several spectra of Quintuplet cluster stars that are virtually "clones" of our group 1 spectra, and none exhibit evidence for features at $2.189 \mu \mathrm{m}$. Shara et al. (2009) display a $K$-band spectrum of the known W-R 102d, classified as WN9, that equally lacks He II $2.189 \mu \mathrm{m}$. Finally, our spectra at both $H$ and $K$ band resemble the Ofpe/WN9 stars of Morris et al. (1996; their Figures 2 and 3). Only some of those stars exhibit He II $2.189 \mu \mathrm{m}$. However, the Of spectra of Hanson et al. (1996) demonstrate that He II $2.189 \mu \mathrm{m}$ is always present for spectral types earlier than O9. Incidentally, the Oe spectra of Hanson et al. (1996) are very similar in appearance to our group 1 stars and do not show He II $2.189 \mu \mathrm{m}$. While the exact classification of these types of spectra is clearly still an open question, they undoubtedly indicate massive stars most likely on their way to a W-R phase. For the purposes of our study, we will label these stars as Oe/WN9.

The final two spectra remaining to be classified are shown in Figure 6, together with our newly discovered WC9 star. Star no. 20 exhibits $\mathrm{Br} \gamma$ emission, He I $2.112 \mu \mathrm{m}$ in absorption, as well as possible $\mathrm{Mg}$ II emission in the $K$ band and $\mathrm{He}$ I $1.70 \mu \mathrm{m}$ absorption and a hint of the $\mathrm{H}$ I series in absorption. Based on the absence of obvious He II features, we classify star no. 20 as type Be. Source no. 28a also shows He I 1.70 and $2.112 \mu \mathrm{m}$ (and 

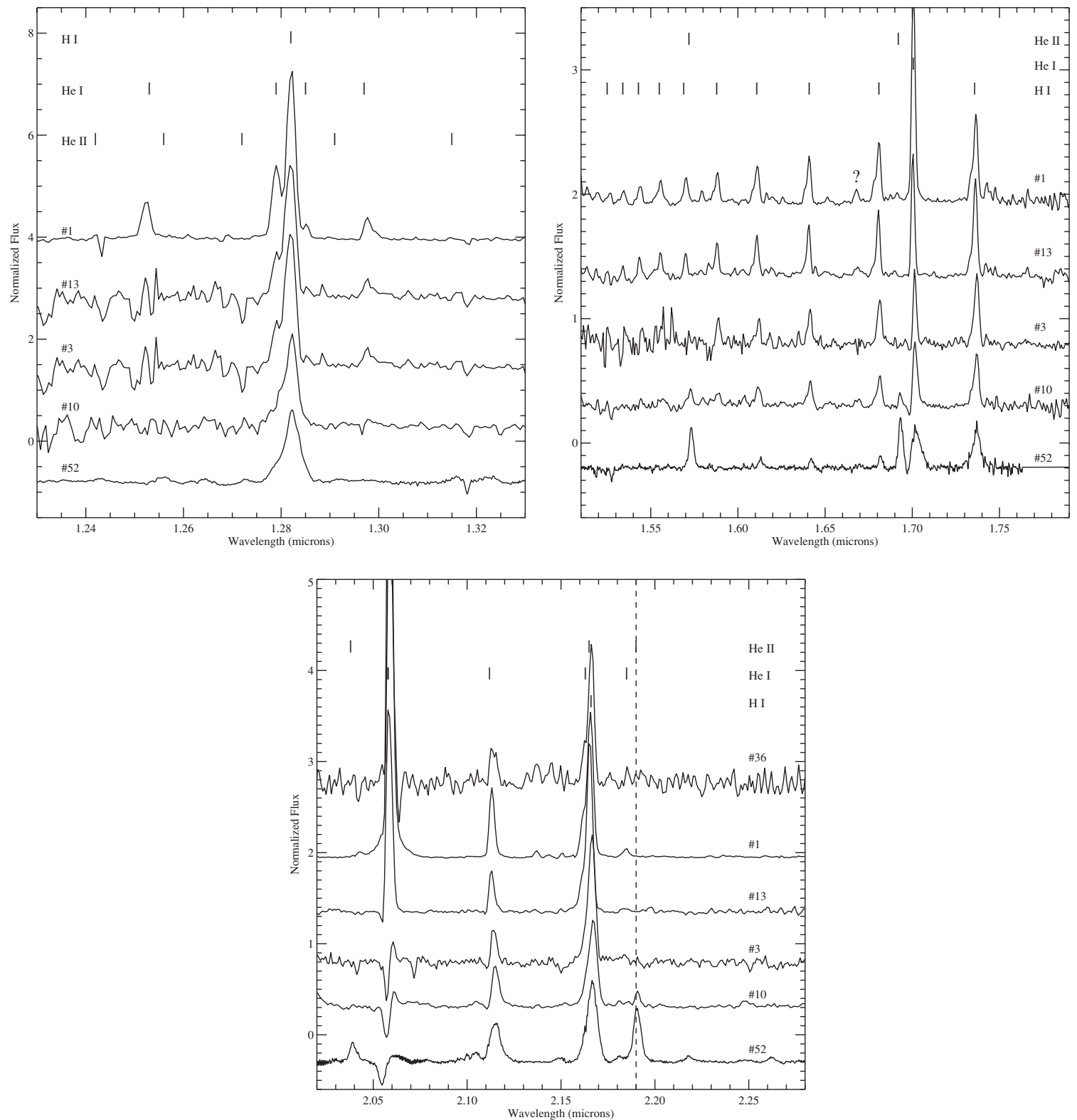

Figure 3. $J-, H$-, and $K$-band spectra of shell central sources characterized by $\mathrm{HI}$ and He I (1.70 and $2.112 \mu \mathrm{m}$ ) emission (group 1$)$. Star no. 10 is a newly discovered WN9h star, star no. 52 a newly discovered WN7 star. The spectra have been normalized and offset from each other for display purposes.

possibly $2.185 \mu \mathrm{m}$ ) in absorption and shallow absorption of $\mathrm{H}$. Based on the $K$-band spectrum and comparison to Hanson et al. (1996), the spectrum of star no. 28a most closely resembles that of HD 168021 (B0 Ib), HD 213087 (B0.5 Ib), and HD 226868 (HMXB, O9.7 Iab) in the relative strength of the features at $\operatorname{Br} \gamma$ and $2.112 \mu \mathrm{m}$. However, in the $H$ band the shallow and broad $\mathrm{H}$ I features indicate luminosity class $\mathrm{V}$ and, together with the strength of He I $1.70 \mu \mathrm{m}$ line, a spectral type of O9-B0.5 (Hanson et al. 1998). We classify star no. 28a as OB, noting that the luminosity class issue still remains to be resolved. The final spectral classification for each source is listed in Table 1.

\subsubsection{Known $L B V s$}

Sources no. 29 (WRAY 17-96 = Hen 3-1453), no. 44 $(\mathrm{G} 024.73+00.69=\mathrm{V} 481 \mathrm{Sct})$, and no. $45(\mathrm{G} 026.47+00.02)$ are identified in SIMBAD as an LBV candidate (Egan et al. 2002), confirmed LBV (Clark et al. 2005), and LBV candidate
(Clark et al. 2003), respectively. All have been found to be surrounded by ring nebulae in the mid-IR with the Midcourse Space Experiment (MSX) (Egan et al. 2002; Clark et al. 2003).

LBVs are generally defined as a class of massive, unstable stars that populate the upper left corner of the H-R diagram (for a detailed review see Humphreys \& Davidson 1994). These high luminosity stars are thought to be a short-lived phase before the $\mathrm{W}-\mathrm{R}$ stage, where a massive star sheds a significant amount of its mass. Consequently, LBVs are characterized by high mass loss rates, significant photometric and spectral variability and occasional mass eruptions, the most famous example being the historic 10 mag brightening of $\eta$ Carinae in the 1840s. These outbursts are extremely energetic; they have been linked to the discovery of rapidly expanding nebulae surrounding LBVs and, in several cases, have previously been mistaken for supernovae in external galaxies (Van Dyk et al. 2000). More common than such giant eruptions, however, is the presence of a circumstellar nebula or shell, indicating more modest, past mass ejection 

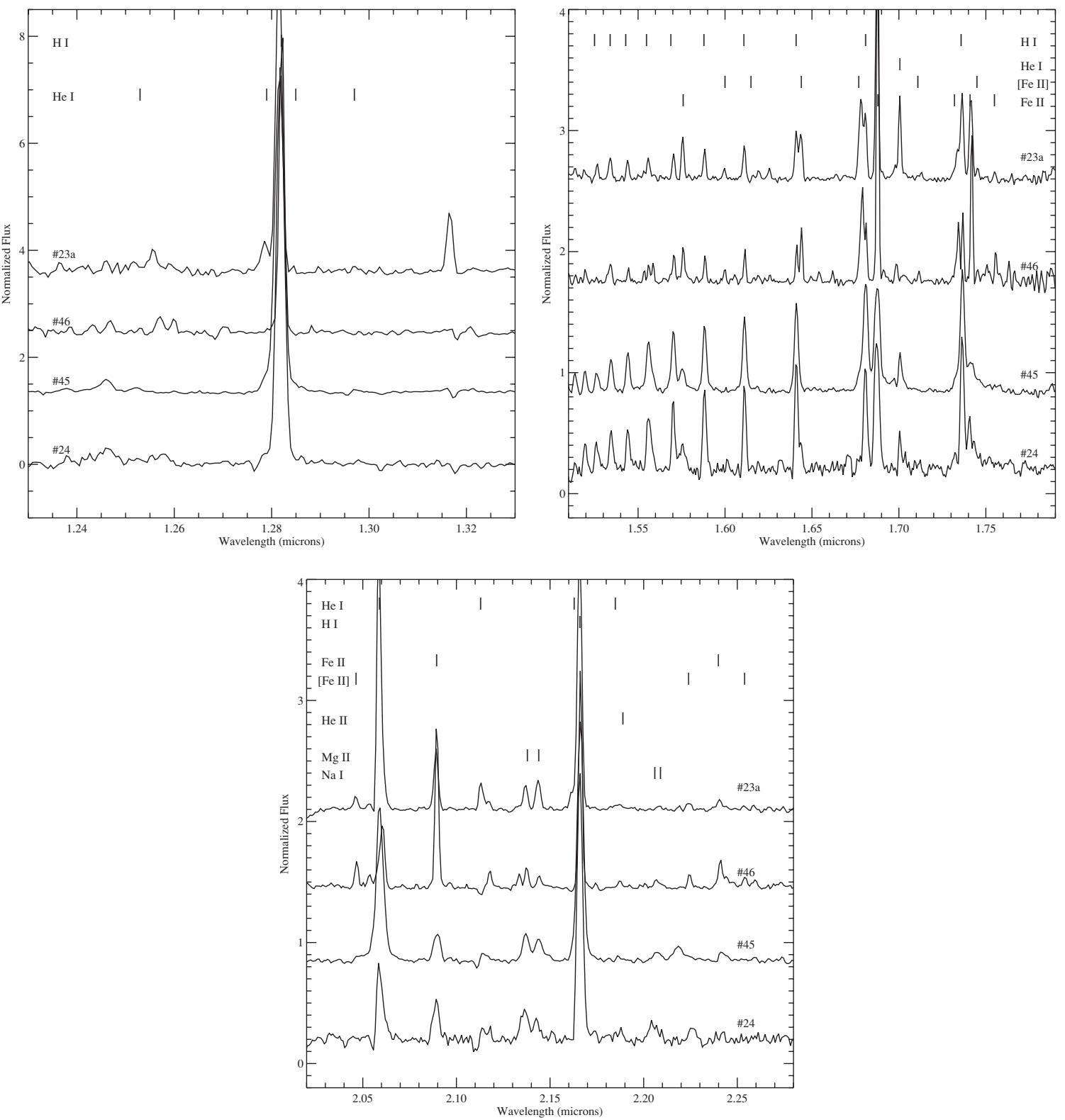

Figure 4. $J-, H$ - and $K$-band spectra of shell central sources characterized by weak or absent He I (1.70, $2.112 \mu$ m) features and strong $\mathrm{HI}$, Fe II, and Mg II emission (group 2A). These sources strongly resemble the stars classified as LBVs and Be/B[e] stars by Morris et al. (1996). The spectra have been normalized and offset from each other for display purposes.

episodes. Out of the 12 confirmed LBVs, seven are associated with circumstellar ejecta, as well as 13 of the $23 \mathrm{LBV}$ candidates listed by Clark et al. (2005). The underlying physical cause that triggers the LBV instability is not yet understood, the current consensus invokes instabilities in the outer layers of the stars as they approach the Eddington luminosity during their evolution.

Our newly acquired spectra of the known LBVs allow us to search for the expected spectral variations in comparison to the spectra previously published in the literature. LBVs in quiescence (at visual minimum) typically exhibit spectral types of $\mathrm{Be}$ or $\mathrm{B}[\mathrm{e}]$, rich with emission lines of hydrogen, helium, iron, and sodium. In many LBVs, the Fe II and [Fe II] emission lines are most pronounced during the quiescent state. During an outburst, the spectra may morph into those of much cooler type $\mathrm{A} / \mathrm{F}$ supergiants as a pseudo-photosphere is formed in the optically thick expanding stellar wind. In one particularly remarkable display, the quintessential LBV S Doradus changed spectral type from B2e to F sometime between 1996 and 1999 (Massey 2000).

Our $K$-band spectrum of WRAY 17-96 does not reveal any significant changes compared to the one presented in Egan et al. (2002), except in the highly variable He I $2.058 \mu \mathrm{m}$ line. To our knowledge our $H$-band spectrum is the first one published of this source. Our $K$-band spectrum of G026.47+00.02 appears identical to that of Clark et al. (2003). Our $H$-band spectrum is of higher resolution and shows additional lines not resolved in the one by Clark et al. (2003).

For G024.73+00.69, our spectra reveal dramatic differences compared to those from 2001/2002 presented by Clark et al. (2003). In our $K$-band spectrum, the strength of the Na I doublet is significantly enhanced with respect to $\mathrm{Br} \gamma$, and the $\mathrm{He} \mathrm{I}$ $(2.112 \mu \mathrm{m})$ line has all but disappeared. In the $H$ band, the changes are even more pronounced as the Hi series has transitioned from emission to absorption. Clark et al. (2003) 

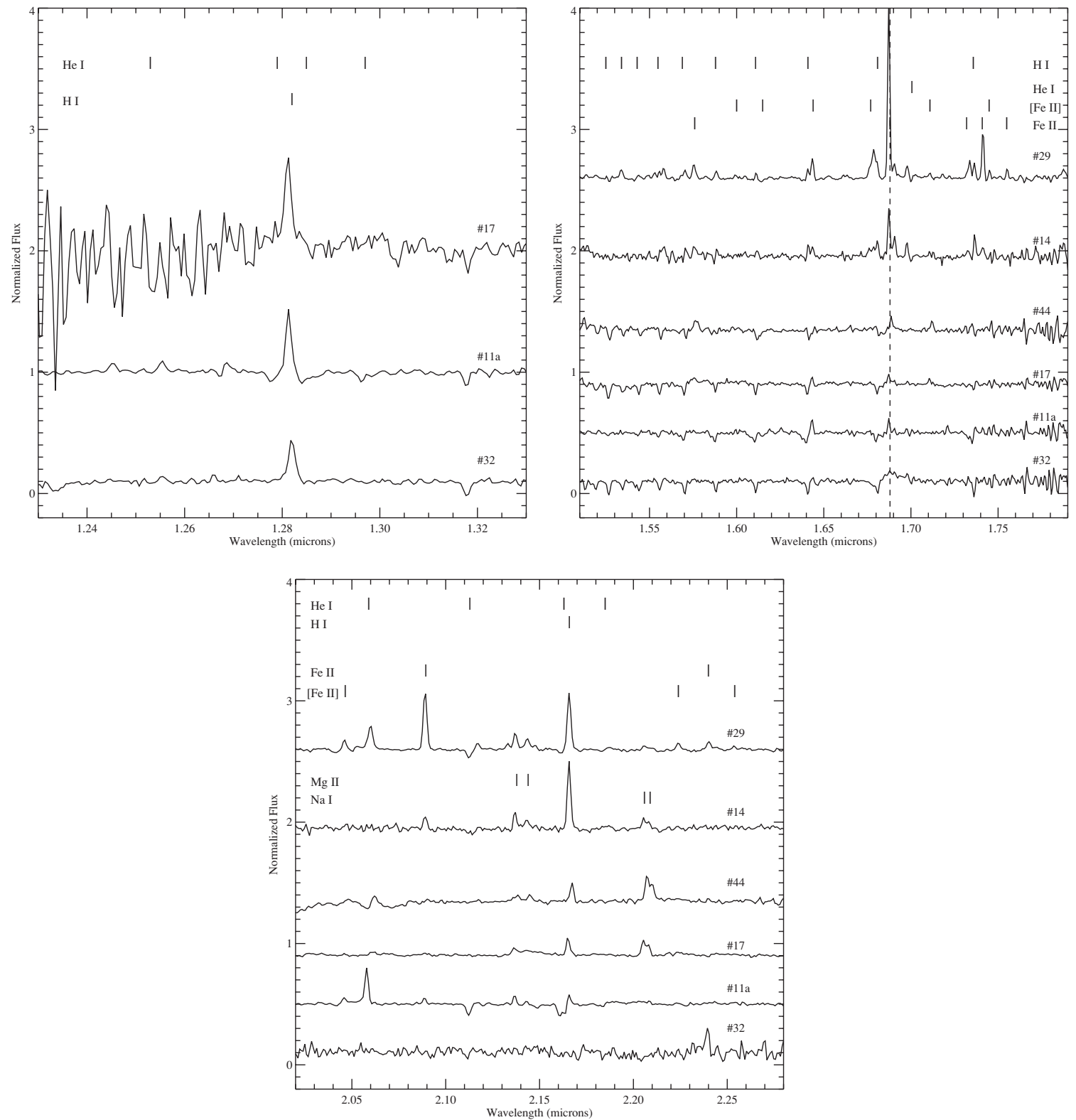

Figure 5. $J$ -,$H$ - and $K$-band spectra of shell central sources characterized by weak or absent $\mathrm{He}$ I $(1.70,2.112 \mu \mathrm{m})$ features and strong Fe II or Mg II emission (group 2B). The spectra are similar to those of group 2A (Figure 4) but exhibit weaker H I features. Note that we could not extract $J$-band spectra for all of these sources. The spectra have been normalized and offset from each other for display purposes.

derived a temperature of $12,000 \mathrm{~K}$ from their $2001 / 2002$ spectra of G024.73+00.69. However, the appearance of our spectra, in particular the absence of He I lines, now implies a significantly lower temperature. This spectral variability, in addition to the photometric variability discovered by Clark et al. (2003), further solidifies the confirmation of G024.73+00.69 as a bona fide LBV.

The new LBV candidates identified in the previous section are strikingly similar in appearance to these known sources. Star no. 24 looks almost identical to no. 45 (G026.47+00.02). Star no. 14 resembles no. 29 (WRAY 17-96) with overall weaker features and star no. 17 is virtually a twin of no. 44 (G 024.73+00.69). Star nos. 46 and 23a stand out due to their pronounced [Fe II] and Fe II emission, and bear a strong resemblance to the spectrum of WRA 751 in the $K$ band (see Figure 8 of Morris et al. 1996).

Not surprisingly, our MIPS $24 \mu \mathrm{m}$ images of the shells (see Figure 1) reveal significantly more detailed structure than the MSX images. The morphologies of the shell nos. 29 and 44 are remarkably similar, showing dense shells with fairly even surface brightness. Both shells are also strongly detected at $8 \mu \mathrm{m}$. Clark et al. (2003) reported the possible detection of a much larger outer shell for no. 44 . The reality of this outer shell is difficult to confirm in our $8 \mu \mathrm{m}$ and $24 \mu \mathrm{m}$ images, given the complex structure in the general background emission evident at both wavelengths. It is equally likely that the areas interpreted as "lobes" by Clark et al. (2003) are associated with unrelated embedded sources visible in the vicinity of that emission. In contrast to shell nos. 29 and 44, shell no.45 displays a more clumpy and filamentary structure. Some faint extended emission enhancements are seen at $8 \mu \mathrm{m}$ coincident with the brighter areas at $24 \mu \mathrm{m}$, but the morphology of the extended emission close to the central star is quite different from that at $24 \mu \mathrm{m}$.

In addition to the known LBV/LBV candidates discussed so far, shell no. 33 of our sample is associated with the LBV candidate HD 316285. We did not obtain any spectra of the central source as part of this work, but Hillier et al. (1998) 

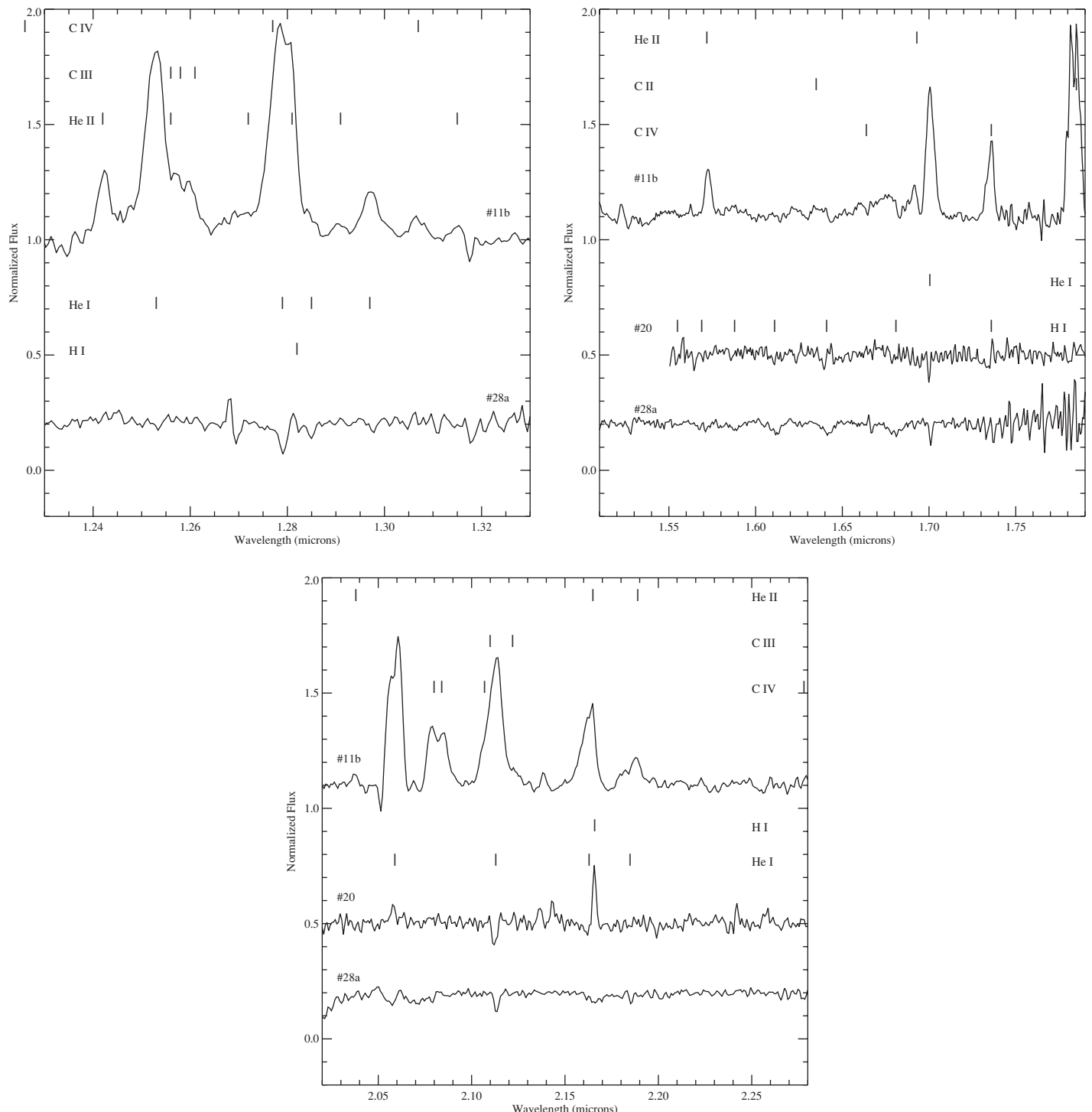

Figure 6. Spectra of the newly discovered WC9 (no. 11b) star as well as the two spectra that did not fit in the classification scheme of the sources in groups 1 and 2 . The spectra have been normalized and offset from each other for display purposes.

present optical and near-IR spectra of the star. Their $H$ - and $K$-band spectra look almost identical to those of our central source no. 45. The star was known to be surrounded by a dusty shell based on the IRAS spectral energy distribution (SED; McGregor et al. 1988). The Spitzer $24 \mu \mathrm{m}$ image of HD 316285 is the first observation to actually resolve the shell. Its morphology, as well as Spitzer mid-IR spectroscopy of the shell, is presented in Morris (2008). Note that there is some extended emission at $8 \mu \mathrm{m}$ as well, however, the morphology of that structure is very different compared to that at $24 \mu \mathrm{m}$. Overall, the characteristics of shell no. 33 are quite similar to those of shell no. 45. Among our newly identified LBV candidates (nos. 11a, 14, 17, 23a, 24, and 46), shells nos. 14, 17, 24, and 46 most strongly resemble the shell morphologies of the known LBVs/LBV candidates, with shell nos. 11 and 46 also exhibiting emission at $8 \mu \mathrm{m}$.

\subsection{Optical Spectra}

Out of the nine central sources observed in the optical regime, two-star nos. 50 and 52-are newly discovered WN stars
(Figure 7). We measured the EW of N IV $\lambda 4057$, the N III $\lambda 4640$ blend, and $\mathrm{Nv} \lambda 4604,4620$ and followed the classification scheme outlined in Conti et al. (1990). In addition, we compared our red spectra to those of the W-R spectral catalog by Vreux et al. (1983). We determine a spectral type of WN6 for source no. 50. Note that this source was previously classified as a planetary nebula (PN), PN G029.0+00.4, its discovery dating back to 1966 (Abell 1966). For star no. 52, we derive a subtype of WN7, confirming the classification obtained earlier from our IR spectrum of this source. We also checked for the presence of hydrogen following the methodology of Smith et al. (1996), and arrive at a classification of WN6o for star no. 50 and WN7(h) for star no. 52

We utilized the spectral atlases of Allen \& Strom (1995) and Torres-Dodgen \& Weaver (1993) for an estimate of the spectral type for the remainder of the central sources. We also checked these spectral types through comparison with observations of spectral type standards obtained on the same nights as the targets. Source no. 59b is an early M-type star, $\sim \mathrm{M} 2-\mathrm{M} 4$ (the spectrum is not shown). The other six source spectra are displayed in Figure 8. In general, the blue spectra are quite 

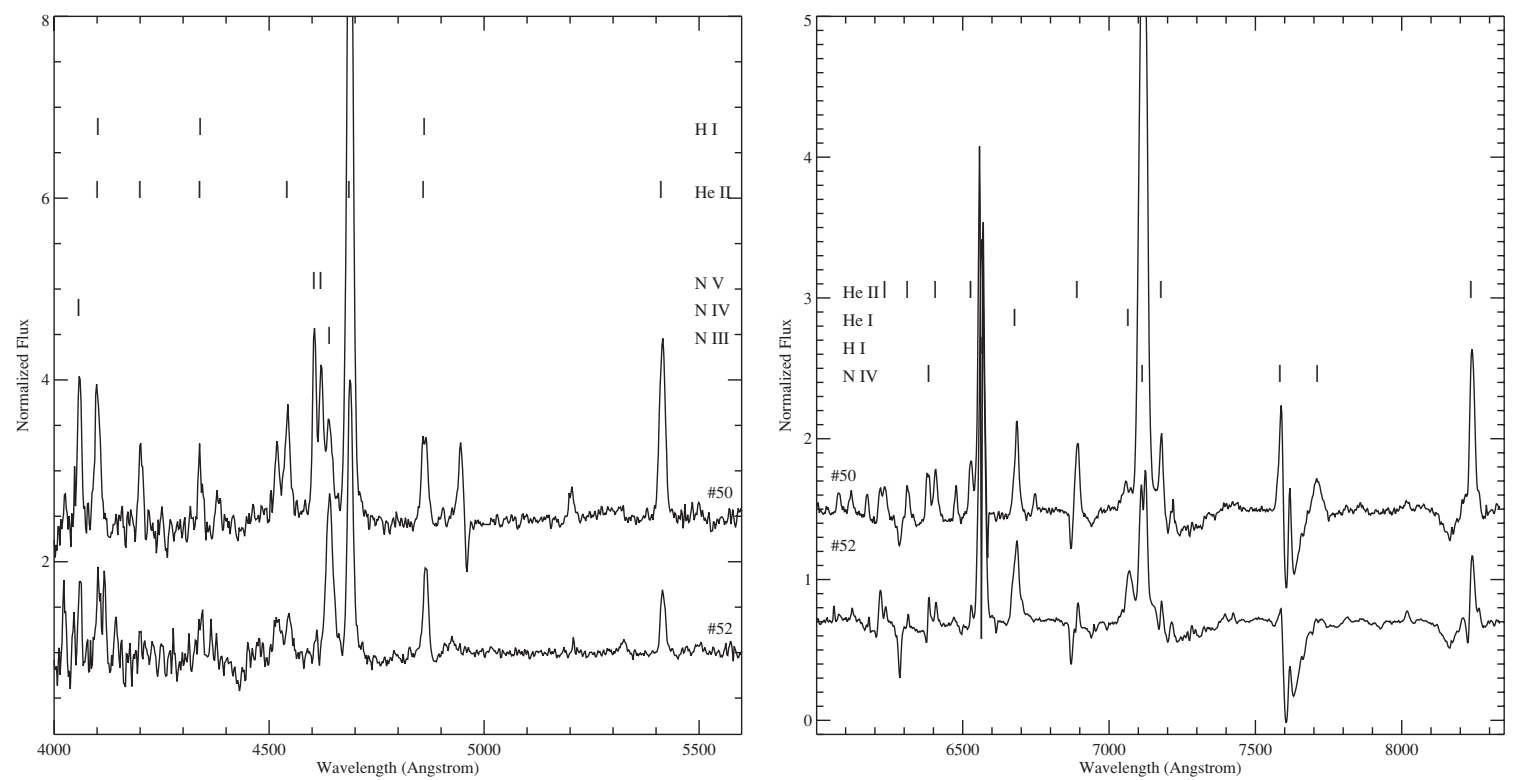

Figure 7. Optical spectra of the newly discovered WN-type W-R star no. 50 (WN6o) and star no. 52 (WN7(h)). The spectra have been continuum normalized and offset for display purposes.

noisy due to the highly reddened nature of these sources. Star nos. 39 and 44 were too faint to extract a signal at the shorter wavelengths, hence only red spectra could be obtained. Source nos. 58, 60, and 61 are classified as B-type stars ( $\sim$ B0-B5) based on the presence of $\mathrm{He}$ I (absorption or emission) and absence of He II $\lambda 4541$ and $\lambda 4686$ (Walborn \& Fitzpatrick 1990). Source no. 60 has a pure $\mathrm{HI}, \mathrm{He}$ I emission spectrum. Source no. 44 is the known LBV G024.73+00.69 discussed in the previous section. Source no. 39 most likely also belongs to the group of B stars, but the red spectrum is quite noisy and it is difficult to gauge the reality of weak absorption features. Star no. 57 most closely matches an F-/G-type spectrum ( $\sim$ F5-G5). We are unable to determine the luminosity class for these sources based on our spectra. Note that the modulation in the continuum of the blue spectra (starting around $5300 \AA$ ) is an artifact of the data reduction process.

\section{INFRARED PHOTOMETRY}

2MASS and GLIMPSE photometry for each source has been assembled in Table 3. Following Mauerhan et al. (2009) and Hadfield et al. (2007), we constructed a color-color diagram (Figure 9) to explore any correlations between the known observed, and still unobserved sources of our sample. The comparison population of sources (black points) is composed of the photometrically most reliable point sources of a representative $1^{\circ} \times 1^{\circ}$ "slice" of the Galactic plane from the GLIMPSE survey. The various types of sources we have identified spectroscopically are indicated by different colors (see the caption of Figure 9 for details).

Given the relatively small sample size for each group, one might expect limited insight from the distribution of points in color space, however, some general trends seem to emerge. As already noted in Mauerhan et al. (2009) and Hadfield et al. (2007), the W-R stars (red points) separate quite cleanly from the main locus of field stars. The W-R stars in our sample form a surprisingly well-defined and tight sequence, roughly parallel to the reddening vector and offset to redder $K s-[8.0]$ colors from the bulk of the distribution of "normal" stars. The direction of the reddening vector at the locus of our W-R stars is indicated by the dashed line. Compared to the analogous figure presented in Mauerhan et al. (2009), about half of our W-R points have larger $J-K s$ values, indicating a more heavily reddened population. B stars and LBV candidates appear to populate the locus in between the normal stars and the W-R track. Obviously, there is some overlap between the location of these different sources in the color-color diagram, since star nos. 24, 44, and 60 lie on the sequence defined by the W-Rs in our sample. Note that there are also some outliers among the F-M group of sources that are on or close to the W-R sequence (star nos. 23b, 26b, and 57). For the late-type stars, this shift to redder colors might indicate a mid-IR excess due to cool circumstellar material. These objects could constitute a significant source of contamination for color-based searches for W-R stars.

Having identified the preferred loci for the various types of central stars, we now turn our attention to the as yet unclassified sources (green points). The reddest outlier at $K s-[8.0]>4$ is source no. 59 a, the bluest at $H-K s \approx 5.2$ is source no. 27 b. Their nature is unclear. Star nos. 41, 47, 48, and 54 are tightly clumped on the W-R sequence at $K s-[8.0] \approx 2.1$ and hence are the best candidates for W-R stars waiting to be identified. Star nos. 2, 25, 34, and 55 are found among the main locus of field stars and most likely belong to the group of F-M type stars. The remainder of the sources of unknown type (with photometry in all bands) are sources nos. 12, 15, 19, 27a, 30, 35, 38, 41, $47,48,49,51,53,54$, and 56 . We expect a mix of W-R, LBV candidates, and F-M type stars among this group, but are unable to make any more finely differentiated predictions.

\section{SHELL MORPHOLOGY, SED, AND SIZE}

Morphologically, our $24 \mu \mathrm{m}$ shells separate broadly into two categories: fainter, more elliptical shells with somewhat irregular rims, and strikingly circular nebulae with sharply defined edges. Shells associated with late-type stars (our F-M types) overwhelmingly belong to the first category (with the exception of shells nos. 8 and 57), while the early-type stars are usually exhibiting the most circular shells (exceptions are nos. $16,18,25$, and 32 ). No distinguishing characteristics are evident in the appearance of the shells between W-R stars, LBV 

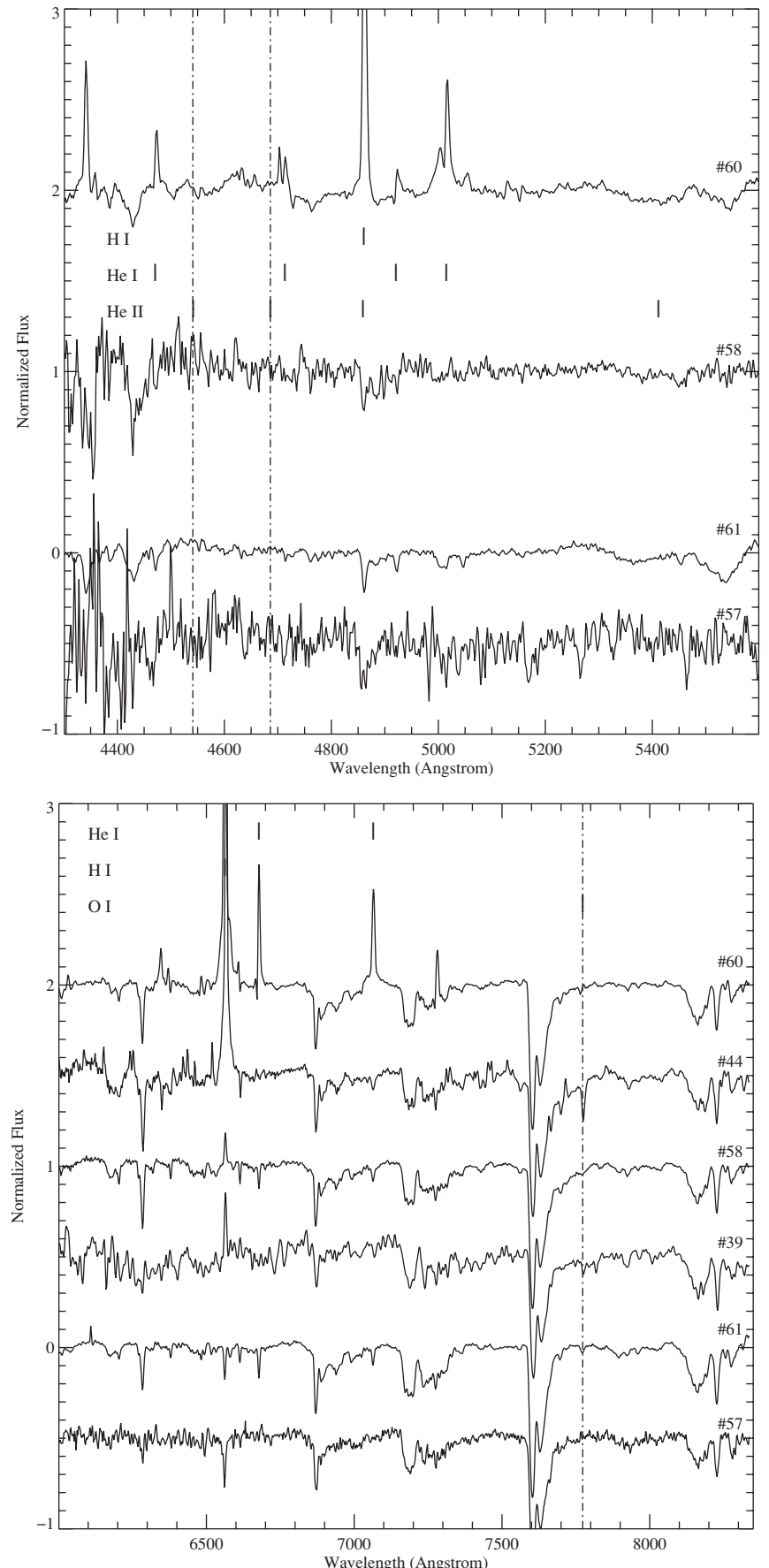

Figure 8. Spectra of the sources classified as B stars (except for source no. 57 which shows a F-/G-type spectrum).

candidates and B stars. The morphology of some of the shells surrounding known LBV stars has already been discussed in Section 4.1.3. Based on the general morphological distinction at $24 \mu \mathrm{m}$ between late- and early-type stars, we conclude that the hot star is likely producing the shell in the case of the double central sources containing both a late- and early-type star (nos. 23 and 28). We also note that the morphology of shell no. 52 provides evidence for multiple, discrete mass loss events. Several concentric rings can be discerned even in the presence of significant structure in the overall background emission.

As noted in Section 1, many of the shells detected at $24 \mu \mathrm{m}$ do not exhibit equivalent emission at $8 \mu \mathrm{m}$ : 37 out of the 61 shells listed in Table $1(60 \%)$ would not have been identified

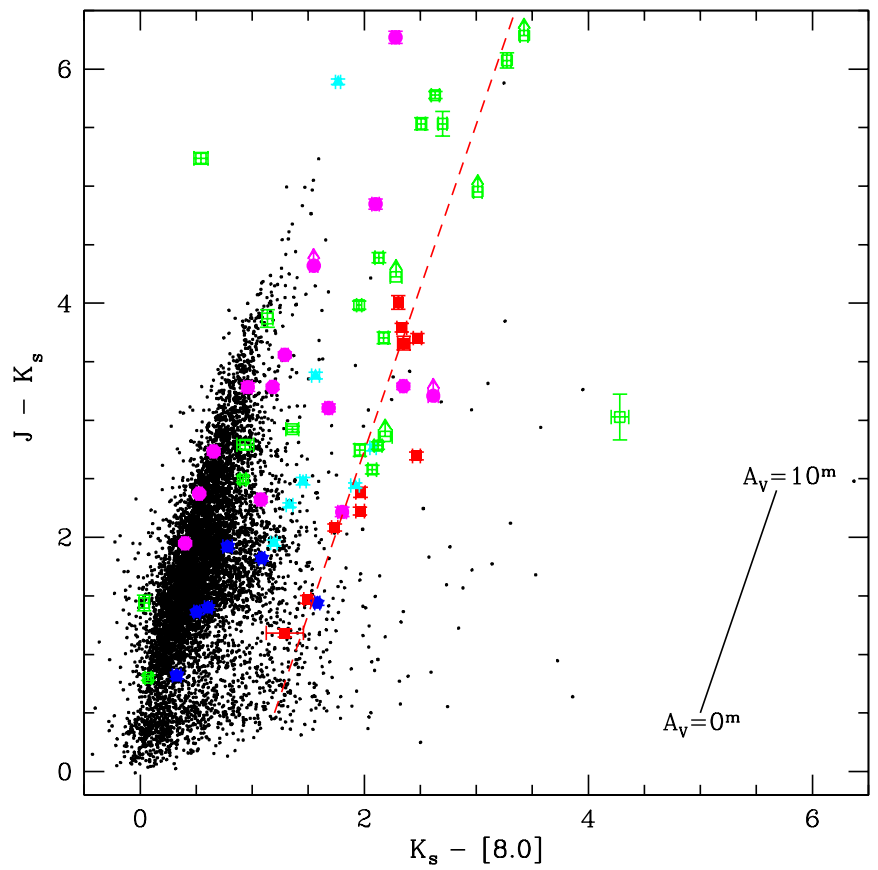

Figure 9. Color-color diagram for the sources in our sample. W-R stars are indicated in red, LBVs/LBV candidates in cyan, B stars in blue, and F-M stars in magenta. Green symbols indicate the central sources that are listed in Table 1, which have not yet been observed spectroscopically. The comparison population of sources (black points) is composed of the photometrically most reliable point sources of a representative $1^{\circ} \times 1^{\circ}$ "slice" of the Galactic plane from the GLIMPSE survey. The reddening vector was adapted from Indebetouw et al. (2005).

as shell sources at $8 \mu \mathrm{m}$. Considering each of the spectral type subcategories, both of the known PNe, five out of the nine latetype stars, none of the B-type stars, two out of the nine Oe/WN and five out of the $10 \mathrm{Be} / \mathrm{B}[\mathrm{e}] / \mathrm{LBV}$ type stars show $8 \mu \mathrm{m}$ shell emission. The mid-IR spectroscopic observations of some LBV and W-R shells imply that the non-detection of $8 \mu \mathrm{m}$ emission could simply be explained by a reduction in flux density at this wavelength relative to that at $24 \mu \mathrm{m}$. The spectra presented by Voors et al. (2000) and Barniske et al. (2008) show a steep increase in emission toward longer wavelength starting at around $10 \mu \mathrm{m}$. At the same time, we cannot exclude the possibility that emission only observed at $24 \mu \mathrm{m}$ might be caused by a strong line feature in the spectrum, such as the [O IV] line producing the shell discovered by Morris et al. (2006).

Based on the general morphological properties of the as yet unclassified sources, we speculate that the shells nos. 2, 12, 25,34 , and 59a are formed by late-type stars. Recall that we predicted sources nos. $2,25,34$, and 55 to likely be stars of type F-M from their location in the color-color diagram, supporting this conclusion. We predict that shells nos. 7, 27, 30, 35, 37, $38,41,47,48,49,51,54$, and 56 are associated with earlytype central stars. Shells nos. 15, 19, 40, 42, 53, and 55 remain ambiguous, partially because none of these have a prominent central source at $24 \mu \mathrm{m}$, unlike all of the other shells.

The observed radii of the $24 \mu \mathrm{m}$ shells listed in Table 1 range from 0.14-2.4 with an average value of $\sim 0$.'7. The physical sizes for the shells surrounding late-type stars have been derived in Section 4.1.1 and are listed in Table 2. We now also estimate the dimensions of the shells around W-R type sources among our sample, adopting the intrinsic colors and absolute $K s$-band magnitudes for the various W-R subtypes presented by Crowther et al. (2006). Utilizing the extinction ratios for the JHKs bands 
Table 4

W-R Distances and Shell Sizes

\begin{tabular}{lcccccc}
\hline \hline Source & Type & $\begin{array}{c}M_{K s} \\
(\mathrm{mag})\end{array}$ & $\begin{array}{c}A_{K s} \\
(\mathrm{mag})\end{array}$ & $\begin{array}{c}A_{V} \\
(\mathrm{mag})\end{array}$ & $\begin{array}{c}d \\
(\mathrm{kpc})\end{array}$ & $\begin{array}{c}\text { Size } \\
(\mathrm{pc})\end{array}$ \\
\hline 9 & WN7 & -4.77 & 1.27 & 11.1 & 9.8 & 3.04 \\
10 & WN9h & -5.92 & 2.32 & 20.3 & 7.8 & 1.60 \\
$11 \mathrm{~b}^{\mathrm{a}}$ & WC9 & $-6.10^{\mathrm{b}}$ & 1.70 & 14.9 & 3.8 & 1.73 \\
16 & WN5b & -4.77 & 2.09 & 18.3 & 6.4 & 1.89 \\
50 & WN6 & -4.41 & 0.65 & 5.7 & 16.5 & 1.39 \\
52 & WN7(h) & -4.77 & 0.66 & 5.8 & 5.2 & 2.72 \\
\hline
\end{tabular}

Notes.

${ }^{a}$ Possibly not producing the shell, see the text for details.

b No $M_{K s}$ is given for WC9 stars in Table A1 of Crowther et al. (2006). We adopt the absolute magnitude of star E from their Table 9.

from Indebetouw et al. (2005), we first calculate an average extinction $A_{K s}$ based on the observed 2MASS $J-K s$ and $H-K s$ colors (provided in Table 3). This extinction is combined with the observed $K s$ magnitude and the assumed $M_{K s}$ for the appropriate W-R subtype, and we thus arrive at the distances and physical shell sizes listed in Table 4. Note that in addition to our newly identified W-R star nos. 10, 11b, 50, and 52, we also list the known W-R star no. 9 (Mauerhan et al. 2009) and star no. 16 (Shara et al. 2009). The shells around these two sources have not been noticed previously, so the physical size estimate for the shells are new calculations, while the distances and $A_{K s}$ values for these stars were already presented in their respective discovery references. The WC9 star no. $11 \mathrm{~b}$ is one of the two possible central sources for shell no. 11 (the fitted center of the shell lies in between the positions of the two stars; see the Appendix). We classify the other star, no. 11a, as belonging to the group of $\mathrm{Be} / \mathrm{B}[\mathrm{e}] / \mathrm{LBV}$ type stars, hence either star could be producing the observed shell, although circumstellar nebulae are more common to $\mathrm{B}[\mathrm{e}] / \mathrm{LBV}$ stars than the more highly evolved WC stars. The close association of two such rare sources makes it likely that they are located at the same distance and could indicate the presence of a small cluster of stars. An investigation of the surrounding sources might be worthwhile. In any case, a distance estimate for one of the sources will determine the physical size of the shell. Unfortunately, Crowther et al. (2006) do not provide a $M_{K s}$ for WC9 stars, only intrinsic IR colors. We have therefore adopted the $M_{K s}$ magnitude of star E from their Table 9 as a rough estimate.

The extinction, distance, and size scales listed in Table 4 seem reasonable, except in the case of star no. 50, where a large distance $(d=16 \mathrm{kpc})$ is paired with a relatively low extinction value $\left(A_{V}=5.7\right)$. This is one of the few objects that exhibits shell emission at optical wavelengths. As already mentioned, the shell was classified as a planetary nebula, but we were unable to find any previous classification of the central star (see Section 4.2 and the Appendix).

Comparing the radii of our W-R shells to those reported in the literature, our shells generally fit well within the observed range, possibly somewhat skewed to the smaller end of the distribution. An optical study of eight W-R ring nebulae by Gruendl et al. (2000) finds radii between 2.1 and $9.0 \mathrm{pc}$, and Chu (1991) quotes a few to $30 \mathrm{pc}$ as the typical diameters of W-R shells. Marston (1996) surveyed 156 W-R stars with IRAS and finds 49 "probable or suspected shells" ranging in size between 5 and $150 \mathrm{pc}$ in radius. Due to the low spatial resolution of IRAS, these detections are clearly biased toward the largest shells.
We are unable to determine the physical sizes for the remainder of our shells, mostly comprising B/Be/B[e]/LBV type stars. It is problematic to derive accurate distances for field $B$ supergiants because they lack luminosity diagnostics in their spectra. Detailed modeling of the stellar IR spectra and shell properties which could constrain the luminosity and reddening of the stars, and determine shell parameters such as mass and dust composition, are beyond the scope of this paper and will be addressed in future work. Clark et al. (2003) summarize the best estimates for the radii of LBV and candidate LBV shell nebulae, which range from 0.1 to $3.6 \mathrm{pc}$, most of them smaller than $\sim 1 \mathrm{pc}$.

\section{SUMMARY AND DISCUSSION}

We have discovered a large number of prominent, highly symmetric $24 \mu \mathrm{m}$ shells surrounding bright central sources. We were able to determine the nature of the apparent central sources for 45 of the 62 shells, 10 based on literature searches and 35 from dedicated spectroscopic follow-up. Our infrared and optical spectroscopy has revealed a mix of early and late-type stars. However, the prevalence of rare, massive stars stands out. We find six bona fide W-R stars in our sample (four of which are new identifications), as well as four stars which we tentatively classify as Oe/WN. Most strikingly, our sample of shells contains four known LBV-type stars (one confirmed, three candidates), and we classify an additional six sources as LBV candidates based on the similarities between the spectra. We realize that the classification of a particular source as an LBV involves a host of criteria, including photometric and spectroscopic variability, that still need to be investigated for these new sources. However, given the small numbers of known LBVs, the presence of a shell combined with the spectroscopic characteristics make this relatively large sample of new candidates an exciting discovery. We also identify five new Be stars in our sample. The distinction between these and what we characterize as LBV candidates is somewhat arbitrary and simply conveys less peculiar spectral properties. It is entirely possible that these stars harbor additional LBV candidates, especially considering LBV spectral variability and the fact that the LBVs AG Car and HR Car have been classified as Be stars.

The detailed evolutionary sequence for the most massive stars is still a matter of intense debate. The standard model of massive star evolution (see, e.g., the review by Crowther 2007) expounds that $\mathrm{O}$ stars with initial masses of $25-40 M_{\odot}$ will undergo an LBV and RSG phase on their way to becoming a W-R star. More massive stars, on the other hand, are thought to avoid the RSG phase. Smith \& Owocki (2006) contend that substantial mass loss during an LBV stage is critical to the formation of W-R stars. Analysis of the nebulae surrounding the known LBVs support the view that the nebulae formed during a BSG and not during an RSG phase (Lamers et al. 2001). Smith \& Conti (2008) strongly argue in favor of the notion that H-rich WN-type stars precede the LBV stage, while H-poor WN stars represent a post-LBV evolutionary phase. In this context, we point out that our Oe/ WN classified sources all show strong $\mathrm{H}$ emission, and hence could be interpreted as pre-LBV stars. In fact, we speculate that a large number of the central stars associated with our most well-defined, circular shells represent a narrow evolutionary phase, closely related or identical to the LBV phase. In depth analysis of the shell emission is needed to further investigate this hypothesis. Finally, we predict that our upcoming observations of the remainder of our sample will reveal additional members of this pre-LBV/LBV group among the as yet unclassified shell central stars. 
This work is based in part on archival data obtained with the Spitzer Space Telescope, which is operated by the Jet Propulsion Laboratory, California Institute of Technology under a contract with NASA. Support for this work was provided by an award issued by JPL/Caltech. Based on observations obtained at the Hale Telescope, Palomar Observatory, as part of a continuing collaboration between the California Institute of Technology, NASA/JPL, and Cornell University. This publication makes use of data products from the Two Micron All Sky Survey, which is a joint project of the University of Massachusetts and the Infrared Processing and Analysis Center/California Institute of Technology, funded by the National Aeronautics and Space Administration and the National Science Foundation. It also utilized NASA's Astrophysics Data System Abstract Service and the SIMBAD database operated by CDS, Strasbourg, France.

Note added in proof: It has been brought to our attention that Gvaramadze et al. (2010) describe optical observations of our source no. 52. Their W-R classification agrees with ours.

\section{APPENDIX}

\section{NOTES ON INDIVIDUAL OBJECTS}

1. IRAS 11419-6228 is coincident with the central source position.

2. MO 1-184, classified as a OB- star by Muzzio \& Orsatti (1977), is offset by $35^{\prime \prime} .18$ from our central source position and has a $24 \mu \mathrm{m}$ counterpart at the edge of the shell, coincident with an emission enhancement in the shell. Hence, MO 1-184 is distinct from the central source associated with shell no. 2 . Note that the $24 \mu \mathrm{m}$ central source is offset from the IRAC 3.6 and $8.0 \mu \mathrm{m}$ source. In the 2MASS images, several blended sources are visible at the central source position. The 2MASS source listed in Table 1 corresponds to the source that coincides with the brightest IRAC source within $1^{\prime \prime}$ of the center position.

4. IRAS $14173-6124$ is offset by $21^{\prime \prime}$ away from the central source, but is likely associated with the central source. At 2MASS wavelengths, there is a group (cluster?) of bright stars that coincides with the southeastern border of the shell.

5. IRAS 15039-5806 is 64" away and clearly associated with a different bright $24 \mu \mathrm{m}$ point source. In $2 \mathrm{MASS}$, several sources are blended at the central source position.

6 . The fitted geometric center of the ellipse falls halfway between the two bright $24 \mu \mathrm{m}$ sources.

8. IRAS $15293-5602$ is offset by $32^{\prime \prime}$ from the central source and clearly associated with a bright $24 \mu \mathrm{m}$ source on the east rim of the shell. There are four bright $24 \mu \mathrm{m}$ sources within the shell, source no. $8 \mathrm{a}$ is at the geometric center, but we also obtained a spectrum of the source immediately to the west (no. 8b).

9. This W-R star was identified based on 2MASS and GLIMPSE photometric selection criteria by Mauerhan et al. (2009). The presence of the shell, however, has not been noticed previously.

10. IRAS 15421-5323.

11. 2MASS J15491137-5508516 (= IRAS 15452-5459), $260^{\prime \prime}$ distant, is classified as a post-AGB star (proto-PN) in SIMBAD. It is associated with an extremely bright $24 \mu \mathrm{m}$ point source in the MIPSGAL images, but not with our shell. Sahai et al. (2007) present Hubble Space Telescope imaging of the post-AGB star which clearly shows a double lobed structure. However at $24 \mu \mathrm{m}$ there is no large-scale extended emission associated with that source, instead only our shell stands out. The fitted geometric center of the circular shell falls halfway between the two $24 \mu \mathrm{m}$ sources listed in Table 1.

12. IRAS $15517-5334$ is $40^{\prime \prime}$ offset from our shell central source, but lies within the shell. The association is uncertain.

14. IRAS $16254-4739$ (15" offset) clearly associated with our shell. The only references for this object are van der Walt et al. (1996) and Bronfman et al. (1996), who search for 6.7 $\mathrm{GHz}$ methanol maser emission associated with IRAS sources selected to meet criteria for being ultracompact $\mathrm{H}$ II regions. No maser emission was detected for IRAS $16254-4739$.

15. An association with IRAS 16278-4808 (28" away) is possible, but somewhat ambiguous. C* 2338, a carbon star with a position offset by $122^{\prime \prime}$ is clearly associated with another $24 \mu \mathrm{m}$ source.

16. Shara et al. (2009) list a WN5b star (Shara 1093_53) near the center of this shell, which is difficult to pinpoint due to its diffuse outer edge. They did not detect the shell itself, and there are three bright sources within about $8^{\prime \prime}$ of the central region. This is one case where selecting the source coincident with the brightest central $24 \mu \mathrm{m}$ source would have identified the wrong star, as the WN5 star is not associated with any enhanced $24 \mu \mathrm{m}$ emission. We note that IGR J16320-4751, an embedded high mass X-ray binary, is also found in the vicinity (157" offset).

18. IRAS 16396-4555, EM* VRMF 115, Hen 2-179, WRAY 16-232, SS73 63, has been classified as anything from PN to "not a PN," highly obscured Be star to M supergiant, or peculiar emission line star. A good summary of the literature on this source, as well as an optical spectrum, are presented in Pereira et al. (2003). Pereira et al. (2003) classifies the source as a reddened Be star. Note that the shell has a highly clumped morphology.

19. IRAS $16426-4504$ (60" offset) is clearly associated with another $24 \mu \mathrm{m}$ source. IGR J16465-4507 is located nearby $\left(215^{\prime \prime}\right)$, but not coincident with our shell. The IGR source consists of a compact object and a blue supergiant companion of spectral type O9.5Ia (Nespoli et al. 2008).

21. The planetary nebula IC 4637 is discussed as a possible containing a physical double central source with a K4 companion at a separation of 2".42 by Ciardullo et al. (1999). 2MASS clearly shows two sources, but only one appears in the 2MASS Point Source Catalog. The GLIMPSE morphology of the PN shell is described in Phillips \& Ramos-Larios (2008).

22. IRAS 17039-3952 is offset by $30^{\prime \prime}$ located halfway between our shell and another embedded source. The association is ambiguous.

23. IRAS 17050-3921.

26. HD 317949, classified as a K0 star in SIMBAD, is offset by $36^{\prime \prime}$ and definitely associated with another source at $24 \mu \mathrm{m}$ at the edge of the shell. The geometric center of the shell is located between the two $24 \mu \mathrm{m}$ sources.

27. No. 27a is the source closest to the geometric center of the shell. At 2MASS wavelengths, the central two sources appear to be blended with additional sources.

28. The radio source GPA 357.18-0.14 (Langston et al. 2000) is found at a separation of $24^{\prime \prime}$. It is not associated with our central source, but located on the SW rim of the shell. Source no. 28a is closest to the geometric center of the shell. 
29. The LBV candidate WRAY 17-96 was originally thought to be a PN. Egan et al. (2002) describe the detection of a ring nebula with $M S X$ and show a $K$-band spectrum of the central source.

31. Planetary Nebula PN G003.5+02.7, discovered by Boumis et al. (2003) and independently by the MASH survey (Parker et al. 2006). Morphologically, the $24 \mu \mathrm{m}$ shell strongly resembles the $\mathrm{H} \alpha$ shell from the MASH survey. Our identification of the central source is uncertain due to a slight offset of the selected star from the geometric center of the shell.

32. IRAS 17408-3027. Raharto et al. (1984) list [RHI84] 10469 at the position of our central source and give a M2: type. No additional information is provided.

33. HD 316285 is a known LBV candidate. Hillier et al. (1998) present optical and near-IR spectra of the central source. The star is known to be surrounded by a dusty shell based on the IRAS SED (McGregor et al. 1988). The MIPS $24 \mu \mathrm{m}$ image is the first observation to resolve the shell. Note that there is some extended emission at $8 \mu \mathrm{m}$ as well, however, the morphology of that structure is very different from that at $24 \mu \mathrm{m}$.

36. At 2MASS wavelengths, the central source is clearly a blend of two sources. However, only one source is listed in the 2MASS catalog. The location of the source is within the coverage area of the MAGPIS survey (Helfand et al. 2006), however, no radio emission is detected from either the shell or the central source.

41. The X-ray source XGPS-I J182833-102652 is located 118" away, but clearly associated with a different $24 \mu \mathrm{m}$ source and nebulosity. At 2MASS wavelengths, the central source is blended with at least one other star. The location of the source is within the coverage area of the MAGPIS survey, however, no radio emission is detected from either the shell or the central source.

44. G $024.73+00.69$ (=V481 Sct) is a known LBV (Clark et al. 2005 ) with a known shell. A $K$-band spectrum is shown in Clark et al. (2003). No radio emission is detected from either the shell or the central source with the MAGPIS survey.

45. G 26.470+0.021 is a known LBV candidate (Clark et al. 2005) with a known shell. The MAGPIS $20 \mathrm{~cm}$ image shows bright emission coincident with the central source, as well as some nebular structure.

47. Source nos. 47 and 48 form a spectacular complex of two neighboring shells at $24 \mu \mathrm{m}$. We treat each shell component as a separate entity, although the combined structure is also reminiscent of a double lobed nebula. However, there is no obvious central source at the "waist" of this potential bipolar emission. It is unclear whether the two shells are interacting with one another or simply are a chance superposition of two independent shells. Due to the large size of each shell, the identification of the central sources is uncertain. At radio wavelengths, the outline of shell no. 48 is faintly visible in the MAGPIS $20 \mathrm{~cm}$ image, as well as a bright point source near the southern edge of the shell.

49. Both the shell and the central source are visible in the MAGPIS $20 \mathrm{~cm}$ image.

50. This source is identified as planetary nebula PN G029.0+00.4 in SIMBAD. Its discovery dates back to Abell (1966) where it is listed as A48. Condon \& Kaplan (1998) include the object in their catalog of PN in the NRAO VLA sky survey, but note it as "Confused by complex background on the NVSS image." We were unable to find any previous classification of the central star, e.g., no central star type is listed in Zuckerman \& Aller (1986). The shell, but not the central star, is a strong radio source and clearly defined in the MAGPIS image.

51. SIMBAD lists [BSM2002] 18431-0312 4, a cloud of unknown nature at a distance of $53^{\prime \prime}$. This is also IRAS $18431-0312$ which in the $24 \mu \mathrm{m}$ MIPSGAL image is clearly associated with a different object, distinct from our shell. No radio emission is detected from either the shell or the central source with the MAGPIS survey.

56. IRAS $18588+0350$ is offset by $43^{\prime \prime}$, the association is uncertain. No radio emission is detected from either the shell or the central source with the MAGPIS survey.

59. Source no. 59a is closest to the geometric center of the shell. No radio emission is detected from either the shell or the central source with the MAGPIS survey.

60. This source is listed as IRAS $19425+2411$ and HBHA 2203-01 in SIMBAD. The only reference (Kohoutek \& Wehmeyer 1999) simply includes the central source in a catalog of $\mathrm{H} \alpha$ emission line stars. No additional information is provided.

62. The central star of this known PN, NGC 6842, is a blend of two sources at 2MASS wavelengths.

\section{REFERENCES}

Abell, G. O. 1966, ApJ, 144, 259

Allen, L. E., \& Strom, K. M. 1995, AJ, 109, 1379

Barniske, A., Oskinova, L. M., \& Hamann, W.-R. 2008, A\&A, 486, 971

Benjamin, R. A., et al. 2003, PASP, 115, 953

Boumis, P., Paleologou, E. V., Mavromatakis, F., \& Papamastorakis, J. 2003, MNRAS, 339, 735

Bronfman, L., Nyman, L.-A., \& May, J. 1996, A\&AS, 115, 81

Cardelli, J. A., Clayton, G. C., \& Mathis, J. S. 1989, ApJ, 345, 245

Carey, S. J., et al. 2009, PASP, 121, 76

Chu, Y. H. 1991, in IAU Symp. 143, Wolf-Rayet Stars and Interrelations with Other Massive Stars in Galaxies, ed. A. Karel (Dordrecht: Kluwer), 349

Ciardullo, R., Bond, H. E., Sipior, M. S., Fullton, L. K., Zhang, C.-Y., \& Schaefer, K. G. 1999, AJ, 118, 488

Clark, J. S., Egan, M. P., Crowther, P. A., Mizuno, D. R., Larionov, V. M., \& Arkharov, A. 2003, A\&A, 412, 185

Clark, J. S., Larionov, V. M., \& Arkharov, A. 2005, A\&A, 435, 239

Condon, J. J., \& Kaplan, D. L. 1998, ApJS, 117, 361

Conti, P. S., Massey, P., \& Vreux, J.-M. 1990, ApJ, 354, 359

Cox, A. N. (ed.) 2000, in Allen's Astrophysical Quantities (4th ed.; New York: Springer)

Crowther, P. A. 2007, ARA\&A, 45, 177

Crowther, P. A., Hadfield, L. J., Clark, J. S., Negueruela, I., \& Vacca, W. D. 2006, MNRAS, 372, 1407

Davies, B., Figer, D. F., Kudritzki, R.-P., MacKenty, J., Najarro, F., \& Herrero, A. 2007, ApJ, 671, 781

Depoy, D. L., Atwood, B., Byard, P. L., Frogel, J., \& O’Brien, T. P. 1993, Proc. SPIE, 1946, 667

Egan, M. P., Clark, J. S., Mizuno, D. R., Carey, S. J., Steele, I. A., \& Price, S. D. 2002, ApJ, 572, 288

Figer, D. F., MacKenty, J. W., Robberto, M., Smith, K., Najarro, F., Kudritzki, R. P., \& Herrero, A. 2006, ApJ, 643, 1166

Figer, D. F., McLean, I. S., \& Morris, M. 1999, ApJ, 514, 202

Figer, D. F., McLean, I. S., \& Najarro, F. 1997, ApJ, 486, 420

Gruendl, R. A., Chu, Y.-H., Dunne, B. C., \& Points, S. D. 2000, AJ, 120, 2670

Gvaramadze, V. V., Kniazev, A. Y., Hamann, W.-R., Berdnikov, L. N., Fabrika, S., \& Valeev, A. F. 2010, MNRAS, 403, 760

Hadfield, L. J., Van Dyk, S. D., Morris, P. W., Smith, J. D., Marston, A. P., \& Peterson, D. E. 2007, MNRAS, 376, 248

Hanson, M. M., Conti, P. S., \& Rieke, M. J. 1996, ApJS, 107, 281

Hanson, M. M., Rieke, G. H., \& Luhman, K. L. 1998, AJ, 116, 1915

Helfand, D. J., Becker, R. H., White, R. L., Fallon, A., \& Tuttle, S. 2006, AJ, 131,2525

Hillier, D. J., Crowther, P. A., Najarro, F., \& Fullerton, A. W. 1998, A\&A, 340, 483

Humphreys, R. M., \& Davidson, K. 1994, PASP, 106, 1025

Indebetouw, R., et al. 2005, ApJ, 619, 931 
Kohoutek, L., \& Wehmeyer, R. 1999, A\&AS, 134, 255

Lamers, H. J. G. L. M., Nota, A., Panagia, N., Smith, L. J., \& Langer, N. 2001, ApJ, 551, 764

Langston, G., Minter, A., D’Addario, L., Eberhardt, K., Koski, K., \& Zuber, J. 2000, AJ, 119, 2801

Marston, A. P. 1996, AJ, 112, 2828

Massey, P. 2000, PASP, 112, 144

Mauerhan, J. C., Van Dyk, S. D., \& Morris, P. W. 2009, PASP, 121, 591

McGregor, P. J., Hyland, A. R., \& Hillier, D. J. 1988, ApJ, 324, 1071

Morris, P. 2008, IAU Symp. 250, Massive Stars as Cosmic Engines, ed. F. Bresolin, P. A. Crowther, \& J. Puls (Cambridge: Cambridge Univ. Press), 361

Morris, P. W., Eenens, P. R. J., Hanson, M. M., Conti, P. S., \& Blum, R. D. 1996, ApJ, 470, 597

Morris, P. W., Stolovy, S., Wachter, S., Noriega-Crespo, A., Pannuti, T. G., \& Hoard, D. W. 2006, ApJ, 640, L179

Muzzio, J. C., \& Orsatti, A. M. 1977, AJ, 82, 345

Nespoli, E., Fabregat, J., \& Mennickent, R. E. 2008, A\&A, 486, 911

Parker, Q. A., et al. 2006, MNRAS, 373, 79

Pereira, C. B., Franco, C. S., \& de Araújo, F. X. 2003, A\&A, 397, 927

Phillips, J. P., \& Ramos-Larios, G. 2008, MNRAS, 383, 1029

Raharto, M., Hamajima, K., Ichikawa, T., Ishida, K., \& Hidayat, B. 1984, Ann. Tokyo Astron. Obs., 19, 469

Rayner, J. T., Cushing, M. C., \& Vacca, W. D. 2009, ApJS, 185, 289
Rieke, G. H., et al. 2004, ApJS, 154, 25

Sahai, R., Morris, M., Sánchez Contreras, C., \& Claussen, M. 2007, AJ, 134, 2200

Shara, M. M., et al. 2009, AJ, 138, 402

Siebenmorgen, R., Natta, A., Kruegel, E., \& Prusti, T. 1998, A\&A, 339, 134

Smith, L. F., Shara, M. M., \& Moffat, A. F. J. 1996, MNRAS, 281, 163

Smith, N. 2002, MNRAS, 336, L22

Smith, N., \& Conti, P. S. 2008, ApJ, 679, 1467

Smith, N., \& Owocki, S. P. 2006, ApJ, 645, L45

Stencel, R. E., Pesce, J. E., \& Bauer, W. H. 1989, AJ, 97, 1120

Torres-Dodgen, A. V., \& Weaver, W. B. 1993, PASP, 105, 693

Vacca, W. D., Cushing, M. C., \& Rayner, J. T. 2003, PASP, 115, 389

van der Walt, D. J., Retief, S. J. P., Gaylard, M. J., \& MacLeod, G. C. 1996, MNRAS, 282, 1085

Van Dyk, S. D., Peng, C. Y., King, J. Y., Filippenko, A. V., Treffers, R. R., Li, W., \& Richmond, M. W. 2000, PASP, 112, 1532

Voors, R. H. M., et al. 2000, A\&A, 356, 501

Vreux, J. M., Dennefeld, M., \& Andrillat, Y. 1983, A\&AS, 54, 437

Walborn, N. R., \& Fitzpatrick, E. L. 1990, PASP, 102, 379

Whitehead, M. J., Meaburn, J., \& Goudis, C. D. 1988, A\&A, 196, 261

Young, K., Phillips, T. G., \& Knapp, G. R. 1993, ApJ, 409, 725

Zuckerman, B., \& Aller, L. H. 1986, ApJ, 301, 772 